\title{
Complicaciones crónicas de la diabetes mellitus: visión práctica para el médico de atención primaria
}

José Antonio Páez'; Javier Dario Triana²; Miguel Ángel Ruiz ${ }^{3}$; Karla Melissa Masmela ${ }^{3}$;esica Alejandra Parada Camila Alejandra Peña ${ }^{3}$; Carlos Mario Perdomo ${ }^{3}$; Raúl Alejandro Quintanilla ${ }^{3}$; Andrés Felipe Ramirez ${ }^{3}$ : Erika Sofía Villamil ${ }^{3}$

1 Médico Internista Fundación Universitaria San Martín, Epidemiología en Universidad del Área Andina, Residente de medicina crítica y terapia intensiva en Fundación Universitaria de Ciencias de la Salud (FUCS).

2 Neurólogo, Clínica de Marly y Hospital San José.

3 Estudiantes semillero de neurología y neurociencias, Facultad de medicina, Fundación Universitaria Juan N, Corpas

2 Médico y cirujano general, especialista en epidemiología y magister en educación.

Correspondencia: Dr. Miguel Ángel Ruiz miguel-ruiz@juanncorpas.edu.co

Como citar: Páez JA, Triana JD, Ruiz MA, Masmela KM, Parada YA, CA Peña, et al. Complicaciones crónicas de la diabetes mellitus. Cuarzo 2016; 22 (1): 13-38.

Recibido: 10 de febrero de 2016. Aceptado: 15 de abril de 2016. Publicado: 30 de junio de 2016.

Licencia creative commons cc)

\section{Resumen}

La diabetes es una enfermedad crónica que se desencadena cuando el páncreas no produce suficiente insulina o cuando el organismo no la puede utilizar con eficacia, derivando en serias complicaciones e incrementando el riesgo de muerte. Según el último informe de la OMS se aprecia un marcado aumento de la carga global de diabetes pasando de 180 millones de personas con esta enfermedad en 1980 a 422 millones en 2014, por lo cual esta organización recomienda mejorar la vigilancia, prevención y control de la diabetes y sus complicaciones. De acuerdo con la metodología, se realizó una búsqueda sistemática en Medline. Las palabras clave empleadas fueron: "Neuropatía diabética", "nefropatía diabética", "diabetes mellitus", "enfermedad cardiovascular" y "pie diabético" En la búsqueda inicial fueron seleccionados 218 artículos originales y de revisión publicados en los últimos cinco años de los cuales 83 cumplían los criterios establecidos, su análisis permitió el desarrollo de un artículo de fácil comprensión enfocado en la fisiopatología y diagnóstico clínico de las complicación anteriormente mencionadas que responde a la problemática mundial en la cual la demanda de pacientes diabéticos supera la oferta de especialistas y en donde el médico de atención primeria juega un papel fundamental en el manejo de estos pacientes.

PALABRAS CLAVE: neuropatía diabética, nefropatía diabética, diabetes mellitus, enfermedad cardiovascular, pie diabético.

\section{Chronic complications of diabetes mellitus: practical vision for the primary care physician}

Diabetes is a chronic disease that is triggered when the pancreas does not produce enough insulin or when the body can'tnot use it 
effectively, leading to serious complications and increasing the risk of death. According to the latest WHO report, there is a marked increase in the global burden of diabetes, from 180 million people with diabetes in 1980 to 422 million in 2014, which is why it recommends improved surveillance, prevention and control of diabetes and its complications. According to the methodology, a systematic search was conducted in Medline. The keywords used were: "Diabetic neuropathy", "diabetic nephropathy", "diabetes mellitus", "cardiovascular disease" and "diabetic foot" In the initial search, 218 original and review articles published in the last five years were selected, of which 83 met the established criteria, their analysis allowed the development of an article of easy understanding focused on the pathophysiology and clinical diagnosis of the aforementioned complications that responds to the global problem in which the demand of diabetic patients surpasses the offer of specialists and In which the primary care physician plays a fundamental role in the management of these patients.

KEYWORDS: Diabetic neuropathy, diabetic nephropathy, diabetes mellitus, cardiovascular disease, diabetic foot.

\section{Introducción}

La diabetes mellitus continúa en aumento y es una de las principales causas de muerte a nivel mundial (1). Según datos publicados para el año 2012 del observatorio colombiano para la diabetes, la tasa más alta reportada de esta enfermedad en Colombia fue de 1851 personas/100.000 hab. en Risaralda, seguida de Santander con 1770 personas/100.000 hab. luego se encuentra Bogotá con 1664 personas/100.000 hab. y Antioquia con 1653 personas/100.000 hab (2). Todos los tipos de diabetes pueden provocar complicaciones en distintas partes del organismo e incrementar el riesgo de muerte, dentro de las principales complicaciones crónicas tenemos: polineuropatía, enfermedad renal crónica, enfermedad cardiovascular, enfermedad cerebrovascular, pérdida de la visión, inmunosupresión entre otras (1). Lo anteriormente expuesto demuestra indiscutiblemente que la DM es una enfermedad con alta prevalencia en Colombia y el mundo cuya demanda según datos del American Diabetes Association (ADA 2016) supera la oferta de especialistas endocrinólogos, internistas, nefrólogos, cardiólogos entre otros, por lo tanto, se plantea la necesidad de que el médico de atención primaria cuente con las competencias necesaria para el abordaje de estos pacientes (3). Este artículo pretende mediante una búsqueda sistemática de la literatura actual, dar al médico de atención primaria una visión práctica de las siguientes complicaciones crónicas asociadas a la DM: polineuropatía, enfermedad cardiovascular y enfermedad renal crónica, con el objetivo de complementar de una forma fácil y rápida la atención de los pacientes diabéticos.

\section{Neuropatía diabética}

\section{Definición}

La neuropatía diabética se define como la presencia de síntomas o signos de disfunción de nervios periféricos en pacientes con diabetes después de la exclusión de otras causas. Se ha dicho que, más que una entidad, es un grupo heterogéneo de enfermedades que presentan un amplio rango de anormalidades. Actualmente la neuropatía diabética es la neuropatía más frecuentemente identificada en clínica, afectando hasta $50 \%$ de los pacientes diabéticos al cabo de 25 años de evolución. La pérdida de sensibilidad protectora causa úlceras de los pies que afectan al 15\% de los diabéticos en algún momento de su vida a lo cual se atribuyen dos tercios de las amputaciones no traumáticas de extremidades inferiores en pacientes con DM (4-7).

\section{Epidemiología}

Según el último informe mundial publicado por la OMS en el año 2016, el número de personas con diagnóstico de DM ha aumentado de 108 millones en 1980 a 422 millones en 2014 y el número de muertes para el año 2012 fue de 1.5 millones (1). En México, se ha reportado que el $60 \%$ de pacientes con diabetes tipo 2 presentan alguna forma de neuropatía diabética y su frecuencia depende de la duración de la diabetes (8). La Asociación Colombiana de Diabetes ha estimado que el $7 \%$ de la población colombiana mayor de 30 años tiene Diabetes tipo 2 y alrededor de un 30 a $40 \%$ de los afectados desconocen su enfermedad (2). 


\section{Fisiopatología}

El enfoque fisiopatológico actual de la neuropatía diabética contempla múltiples causas las cuales se agrupan en aspectos metabólicos (ej: daño oxidativo, osmótico e inflamatorio-autoinmune) y vasculares $(5,8-12)$ :

\section{Aspectos metabólicos}

Los aspectos metabólicos de la fisiopatología se resumen en la figura 1. Con la hiperglicemia, la glucosa que entra al axón y a la célula de Schwann, bajo la acción de la aldosa-reductasa se transforma en sorbitol, produciendo hiperosmolaridad y edema. Además, aumenta la concentración de NADP+ frente a la disminución de NADPH, paralizando la regeneración del glutatión, impidiendo la neutralización de los radicales libres y nitrosilantes, pro- duciendo daño oxidativo. Por otro lado, la mayor oferta de NADH desvía el metabolismo glucídico hacia diacilglicerol (DAG), activador de la proteína-kinasa-C (PKC), la que a su vez induce la producción de los mediadores TGF- $\beta 1$ (transforming-growth factor beta-uno) y NF- $\kappa \beta$ (Nuclear Factor Kappa beta), estimulantes ambos de la inflamación y la fibrosis. Lo anterior explica en parte los fenómenos que conllevan al edema y al estrés oxidativo en los nervios periféricos. Ahora hablaremos de la glicosilación no enzimática de las proteínas en la neuropatía, evento fisiopatológico que reúne características metabólicas e inflamatorias. Existe una reacción en la cual se acumulan proteínas irreversiblemente glicosiladas, denominadas productos de glicosilación avanzada (AGE), en diferentes estructuras del cuerpo humano, esta reacción se conoce como "reacción de Meillard". Esto se intensifica en personas diabéticas, acumulándose en el cristalino del

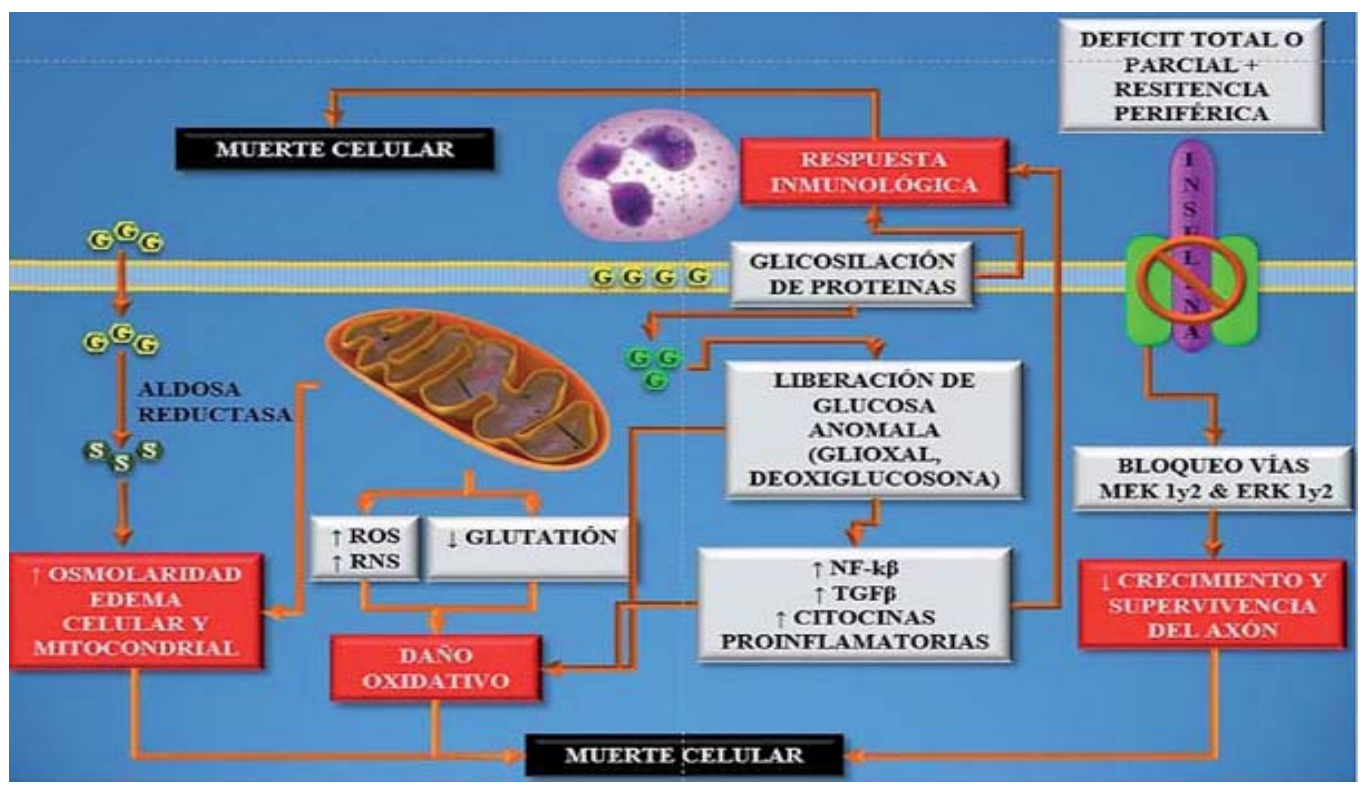

FIGURA 1. Mecanismos metabólicos de la fisiopatología de la polineuropatía diabética. En esta grafica se resumen cuatro importantes mecanismos que llevan a la lesión y apoptosis de las células de Schwann. 1. Daño osmótico producido por el aumento ( $\uparrow$ ) de sorbitol (S) el cual causa edema celular y mitocondrial; 2. Daño oxidativo dado por el aumento $(\uparrow)$ en la concentración de las especies reactivas de oxigeno (ROS) y de nitrógeno (RNS) y al mismo tiempo disminución $(\downarrow)$ de la concentración de glutatión el cual tiene importantes propiedades antixidantes. Este mecanismo oxidativo también se aumenta cuando se liberan sustancias derivadas de la glicosilación avanzada de proteínas, cuando aumentan las sustancias proinflamatorias y cuando disminuye la capacidad de captar glucosa por déficit total o parcial o resistencia a la insulina; 3. Disminución ( $\downarrow$ ) de las señales intracelulares que favorecen la supervivencia y crecimiento del axón dado por resistencia periférica, disminución y en mayor medida bloqueo total de la producción de insulina y 4 . Activación de la respuesta inflamatoria por dos mecanismos principales, el primero es el aumento en la concentración del factor nuclear kappa-beta y el factor de crecimiento transformante beta (NF-k $\beta$ y TGF $\beta$ respectivamente) así como de diferentes interleuquinas proinflamatorias, y el segundo mecanismo depende del reconocimiento de las proteínas glicosiladas que actúan como neoantígenos favoreciendo la destrucción celular mediada por el sistema inmune. Imagen adaptada de: Olmos PR, Niklitschek S, Olmos RI, Faúndez JI, Quezada TA, Bozinovic MA. Bases fisiopatológicas para una clasificación de la neuropatía diabética. Rev Médica Chile. 2012;140(12):1593-1605. 
ojo, el colágeno de las membranas basales y en el componente proteico de la mielina en el sistema nervioso periférico. El proceso de glicosilación de las proteínas puede dividirse en tres fases: iniciación, propagación y finalmente el producto de glicosilación avanzada (AGE) $(5,8-12)$.

En la iniciación, la glucosa reacciona con los residuos amino del aminoácido lisina formando un producto llamado "producto Amadori" (un ejemplo de producto Amadori es la hemoglobina glicosilada), este producto tiene dos destinos posibles: el primero es que si la glucemia se normaliza, entonces este se desglicosila desprendiéndose de la proteína sin dejar daño alguno, sin embargo, la glucosa desprendida pierde sus propiedades y puede convertirse en moléculas de radicales libres violentamente oxidantes (glioxal y 3-deoxiglucosona). Por otro lado, si la hiperglucemia persiste, el producto Amadori sufrirá nuevas oxidaciones en la fase siguiente (fase de propagación). La fase de propagación parte desde la desglicosilación del producto Amadori cuyos resultados son moléculas de radicales libres altamente oxidantes (glioxal y 3-deoxiglucosona), estas moléculas tienen la tendencia a formar productos de glicosilación que como están unidos a una sola proteína, no forman puentes entre ellas (pirralina y N-carboximetil-lisina) $(5,8-12)$.

La fase de la glicosilación avanzada comienza con la unión de la pirralina y la N-carboximetil-lisina con una segunda proteína, formando AGEs no fluorescentes con capacidad de formar puentes, los cuales reciben los nombres de "puentes DOLD" y "puentes GOLD" con lo que se altera definitivamente la estructura terciaria y cuaternaria de las proteínas. Además de los puentes DOLD y GOLD, también se generan AGE que forman puentes entre la lisina de una proteína con la arginina de otra. Entre ellos están el "puente glucosespano" y el "puente pentosidina". En los nervios periféricos, la glicosilación del componente proteico de la mielina hace a ésta apetecible para ser fagocitada por macrófagos que tienen receptores de AGE (RAGE), contribuyendo así, junto con la glicosilación de la tubulina (lo que altera el transporte axonal) a la génesis de la neuropatía diabética. Además de los mecanismos ya expuestos, existen otros mediante los cuales se produce estrés oxidativo adicional, dentro de éstos sobresale el de la auto-oxidación de la glucosa y su relación con la glicosilación. La glucosa en presencia de iones de hierro y cobre y habiéndose transformado en hidroxialdeido (reacción reversible que se produce en presencia de agua) se convierte en un anión radical de enediol, el cual es una molécula inestable que se transforma a su vez en un ketoaldehído como los ya mencionados glioxal y 3-deoxiglucosona produciéndose entonces reducción de la actividad del ciclo de regeneración del glutatión el cual es el principal agente antioxidante de la célula. Cabe resaltar que en el caso de la neuropatía autonómica el mecanismo de daño difiere en ciertos aspectos, una neurona autonómica (simpática) postganglionar típica tiene canales de sodio en la membrana plasmática, en el caso de la DM crónicamente descompensada, el componente alfa del canal de sodio se oxida, de modo que la neurona postganglionar no es capaz de generar sus propios potenciales de acción, esta oxidación se debe a que en condiciones de hiperglicemia, en la neurona autonómica se intensificarían los fenómenos químicos debidos al déficit de NADPH y depleción de glutatión (5,8-12).

Es importante mencionar que la insulina por si misma estimula una serie de factores de crecimiento neuronal que favorecen la reparación y crecimiento del sistema nervioso, en el caso de los pacientes con DM tipo 1 (DM1) esta capacidad se ve afectada ya que hay ausencia de insulina endógena y puede producir manifestaciones tempranas de neuropatía diabética antes que los mecanismos dependientes de hiperglucemia crónica hayan generado el daño suficiente sobre los nervios periféricos. La insulina estimula dos tipos de receptores en la neurona, estos son los Trk-A y P75-NTR, mediante estos receptores la neurona controla como se dijo anteriormente algunos procesos de crecimiento, desarrollo y reparación a nivel del sistema nervioso periférico. Debido a la unión del factor de crecimiento neuronal con el receptor TrkA se activa una cascada de señalización intracelular que estimula las MPP-Kinasas y estas a su vez activan a las vías MEK 1y2 y ERK 1y2, estimulando el crecimiento neuronal. Por su parte las vías que usan RSK vía ERK y, AKT vía PI3-kinasa estimulan la supervivencia neuronal, a través de la activación de Bcl-2 y la inhibición de P53, lo anteriormente nombrado corresponde a la vía del TrkA, por otro lado, la vía del P75-NTR por sí sola podría desencadenar mecanismos de apoptosis neuronal, pero de forma afortunada las señales de esta vía son silenciadas por las de la vía Trk-A. La apoptosis neuronal es estimulada también por la vía MKK4-Bax por la disminución de los factores de crecimiento neuronal que como nombramos antes son activados por la insulina. Desde el punto de vista inflamatorio-autoinmune las neuropatías diabéticas no sólo se limitan a la radículo-plexopatía lumbosacra, sino que también incluyen a la radículo-plexopatía cervical, 
la radiculopatía torácica, y las neuropatías craneales. En estos casos predomina la pérdida axonal y la infiltración leucocitaria que ocurre en este tipo de neuropatías. En la neuropatía diabética de fisiopatología principalmente inflamatorio-autoinmune, el metabolismo intraneuronal de la glucosa se desviaría predominantemente hacia la producción de citokinas proinflamatorias y fibrogénicas como el NF-k $\beta$ (Nuclear Factor Kappa beta) y el TGF $\beta$ (Transforming Growth Factor-beta) (5,8-12).

\section{Aspectos vasculares}

Tradicionalmente se ha dicho que la microangiopatía intervendría en la génesis de la neuropatía diabética. Para debatir acerca de esta hipótesis es necesario tener en cuenta dos aspectos importantes de la circulación de los nervios periféricos, por un lado tenemos que cada nervio periférico cuenta con la irrigación de dos sistemas arteriolares que debido a la localización de sus ramas reciben el nombre de circulación intrínseca y extrínseca; la circulación intrínseca proporciona capilares cuyo flujo en su interior se dirige desde la porción distal hacia la proximal del nervio y la circulación extrínseca proporciona capilares cuyo flujo en su interior se dirige desde la porción proximal hacia la distal. Esta disposición anatómica que además cuenta con múltiples anastomosis entre ambos sistemas asegura que los nervios periféricos cuenten con un adecuado aporte de oxígeno y nutrientes provenientes de la sangre, de modo que resulta difícil que la microangiopatía diabética por si sola pueda producir daño isquémico suficiente en el sistema nervioso periférico que lleve al déficit sensitivo y motor característico de la neuropatía diabética. Por otro lado, existe un aspecto importante en la anatomía de las vénulas que se encuentran en los nervios periféricos que apoyarían la hipótesis vascular, esta teoría de "valvula venosa" se basa en la explicación que cuando una vénula atraviesa el perineuro lo hace en ángulo oblicuo, de modo que un pequeño aumento en la presión del endoneuro produce una obstrucción parcial del flujo venoso limitando la circulación y produciendo isquemia. Este mecanismo adquiere mayor importancia en los nervios que atraviesan canales osteofibrosos inextensibles (ej: canal carpiano y tarsiano) y también los trayectos de nervios craneales $(5,8-12)$.

\section{Cuadro clínico}

Los órganos del cuerpo se ven afectados de forma crónica en la DM por múltiples factores (ej: hiperglucemia crónica, estrés oxidativo, hipoxia tisular, predisposición genética, etc.). Dentro de las principales complicaciones crónicas de la diabetes encontramos aquellas que clásicamente se clasifican como de origen microvascular (ejp: Polineuropatía, la retinopatía, la nefropatía diabética) y macrovascular (ejp: enfermedad coronaria, enfermedad cerebrovascular y enfermedad vascular periférica). En la retinopatía diabética se observa puntos rojos que consisten en pequeños aneurismas de los capilares (microaneurismas) y pequeñas hemorragias causadas por la ruptura de los mismos (microhemorragias). También se pueden observar exudados causados por salida de lipoproteínas (13-15).

La polineuropatía diabética es un grupo de afecciones del sistema nervioso periférico producidas por la DM, estando relacionadas principalmente con su duración y severidad. Ésta puede llegar a producir defectos motores y sensitivos severos al afectar fibras mielínicas gruesas (lo cual genera pérdida de la sensación vibratoria y alteración de la propiocepción) o fibras mielínicas finas (produciendo un deterioro de la sensibilidad dolorosa, el tacto ligero y la temperatura). La presencia de úlceras en los pies es la expresión máxima del compromiso neuropático, determinada por las alteraciones sensitivas, motoras y autonómicas, siendo las úlceras un indicador de neuropatía avanzada, las cuales generan de por sí un importante grado de incapacidad física para el paciente. Para el estudio de la neuropatía es fundamental la realización de una buena historia clínica, una minuciosa exploración física y neurológica dirigida especialmente a la detección de alteraciones sensitivas y motoras, complementada con un examen neurofisiológico general. Las manifestaciones clínicas de la neuropatía diabética dependen del tipo y de la distribución de las poblaciones de nervios afectados, el grado de lesión y el curso de la enfermedad. Existen formas de presentación clínica simétricas y difusas, como la clásica polineuropatía diabética distal de predominio sensitivo (en guante o calcetín), y las formas asimétricas, como las mononeuropatías, lo cual determina un amplio abanico de signos y síntomas. Cuando compromete los nervios motores encontramos debilidad muscular, fatiga, calambres, fasciculaciones, atrofia distal y disminución de los reflejos, si se afectan los nervios sensitivos que conducen el dolor, la temperatura, y el sentido de la posición, habrá disestesias, parestesias, dolor, sensación de quemazón y/o frialdad, hipersensibilidad y ataxia sensitiva y cuando implica disfunción autonómica se encuentra hipotensión arterial postural, alteraciones en el 
tubo digestivo, el sistema urinario, la función respiratoria y cardiovascular, anhidrosis, incontinencia esfinteriana e impotencia sexual (16-18)

\section{Diagnóstico}

Dado que la neuropatía periférica se encuentra dentro de las complicaciones más frecuentes de la DM es fundamental detectarla a tiempo con el objetivo de ofrecer atención temprana. Actualmente se considera que los estudios neurofisiológicos (ejp: medición de la velocidad de conducción nerviosa y electromiografía) son los métodos más objetivos de diagnóstico y seguimiento de esta enfermedad, sin embargo, no se considera su uso de rutina para el diagnóstico de neuropatía en pacientes con DM, sino más bien, deben ser reservados para establecer la gravedad o para cuando existan dudas en cuanto al diagnóstico $(4,6,19)$. Es fundamental que se descarten otras patologías neurológicas que puedan equivocar el diagnóstico (ejp: enfermedad cerebro vascular, tumores del sistema nervio- so, enfermedades desmielinizantes y neurodegenerativas entre otras). Existe una serie de métodos diagnósticos sencillos, enfocados en las manifestaciones clínicas y el examen clínico neurológico de los pacientes, los cuales nos ayudan a establecer la impresión diagnóstica de polineuropatía diabética. Dentro de los más destacados se encuentran:

1. Examen clínico neurológico: el examen clínico neurológico (Tabla 1) es un instrumento compuesto por: 1. Medición de la sensibilidad del pie (percepción de la posición, pinprick test, sensibilidad a estímulos ligeros y vibración), 2. Evaluación de los reflejos en los miembros inferiores, 3. Nivel anatómico por debajo del cual la sensibilidad a estímulos ligeros se encuentra alterada, 4. Fuerza muscular y 5. Reflejo aquiliano. El puntaje máximo es 33 , un puntaje de cero indica ausencia de polineuropatía diabética sintomática, de uno a nueve indica polineuropatía leve, de 10 a 18 moderada y de 19 a 33 polineuropatía severa $(7,20)$.

TABLA 1. Examen clínico neurológico.

ÍTEM

\begin{tabular}{|c|c|c|}
\hline $\begin{array}{l}\text { Nivel por debajo del cual } \\
\text { la sensación está alterada }\end{array}$ & & $\begin{array}{l}0=\text { Normal } \\
1=\text { Dedo del pie } \\
2=\text { Región media del pie } \\
\text { en sentido transverso } \\
3=\text { Tobillo } \\
4=\text { Mitad de la pantorrilla } \\
5=\text { Rodilla }\end{array}$ \\
\hline Fuerza muscular & $\begin{array}{l}\text { Dorsiflexión del pie y } \\
\text { extensión del dedo gordo del pie } \\
\text { Extensión del tobillo }\end{array}$ & $\begin{array}{l}0=\text { Normal } \\
2=\text { Alterado } \\
4=\text { Ausente }\end{array}$ \\
\hline
\end{tabular}

Adaptado de: Yang Z, Chen R, Zhang Y, Huang Y, Hong T, Sun F, et al. Scoring systems to screen for diabetic peripheral neuropathy. En: The Cochrane Collaboration, editor. Cochrane Database of Systematic Reviews [Internet]. Chichester, UK: John Wiley \& Sons, Ltd; 2014 [Consultado 2017 Mar 5]. Disponible en: http://doi.wiley.com/10.1002/14651858.CD010974 
2. Michigan Neuropathy Screening Instrument (MNSI): es un instrumento que incluye dos partes, primero un cuestionario con 15 preguntas (Tabla 2) y segundo (Tabla 3) un examen clínico del pie del paciente. El cuestionario indaga sobre síntomas sensoriales positivos (ejp: dolor, sensación de calor, hormigueo), negativos (ejp: entumecimiento), calambres, debilidad muscular, piel agrietada y amputaciones. Es posible definir el diagnóstico clínico de neuropatía con 7 o más respuestas afirmativas en el cuestionario. Simultáneamente se realiza el examen clínico del pie, éste evalúa la apariencia, presencia de ulceras, reflejo aquiliano y sensibilidad vibratoria mediante el test de diapasón de $128 \mathrm{~Hz}$. El examen físico debe realizarse en ambos pies y el puntaje máximo es 8 , un puntaje mayor o igual a dos es positivo para polineuropatía diabética $(20,21)$.

3. Test del Monofilamento de Semmes-Weinstein (Figura 2): Se trata de un filamento de nailon unido a un mango, que al doblarse aplica una presión constante de 10 $\mathrm{g}$ independientemente de la fuerza con que lo aplique el explorador, evalúa la sensibilidad a la presión táctil lo que se ha denominado sensibilidad protectora. Es uno de los métodos más sencillos y eficaces en la evaluación de la sensibilidad superficial, se usa comúnmente en conjunto con el MNSI y el examen clínico neurológico para elevar la sensibilidad y especificidad. El monofilamento más ampliamente utilizado es el que da una presión de $10 \mathrm{~g}$, ya que, según datos de estudios prospectivos, es el que mejor se correlaciona con la pérdida de la sensibilidad protectora, la presencia de ulceras y con el riesgo de desarrollarlas $(6,20)$.

\section{Tratamiento y prevención}

Existe importante evidencia de que un adecuado control metabólico de la diabetes disminuye la frecuencia y la gravedad de las complicaciones nerviosas periféricas; el objetivo principal del tratamiento es el control estricto de los niveles de glucosa en sangre, así mismo es importante realizar la corrección de anomalías metabólicas (ej: trastornos tiroideos, renales y hepáticos), control nutricional regular, manejo antibiótico adecuado para las complicaciones infecciosas, corrección de posibles agentes agre-

TABLA 2. Cuestionario polineuropatía (MNSI).

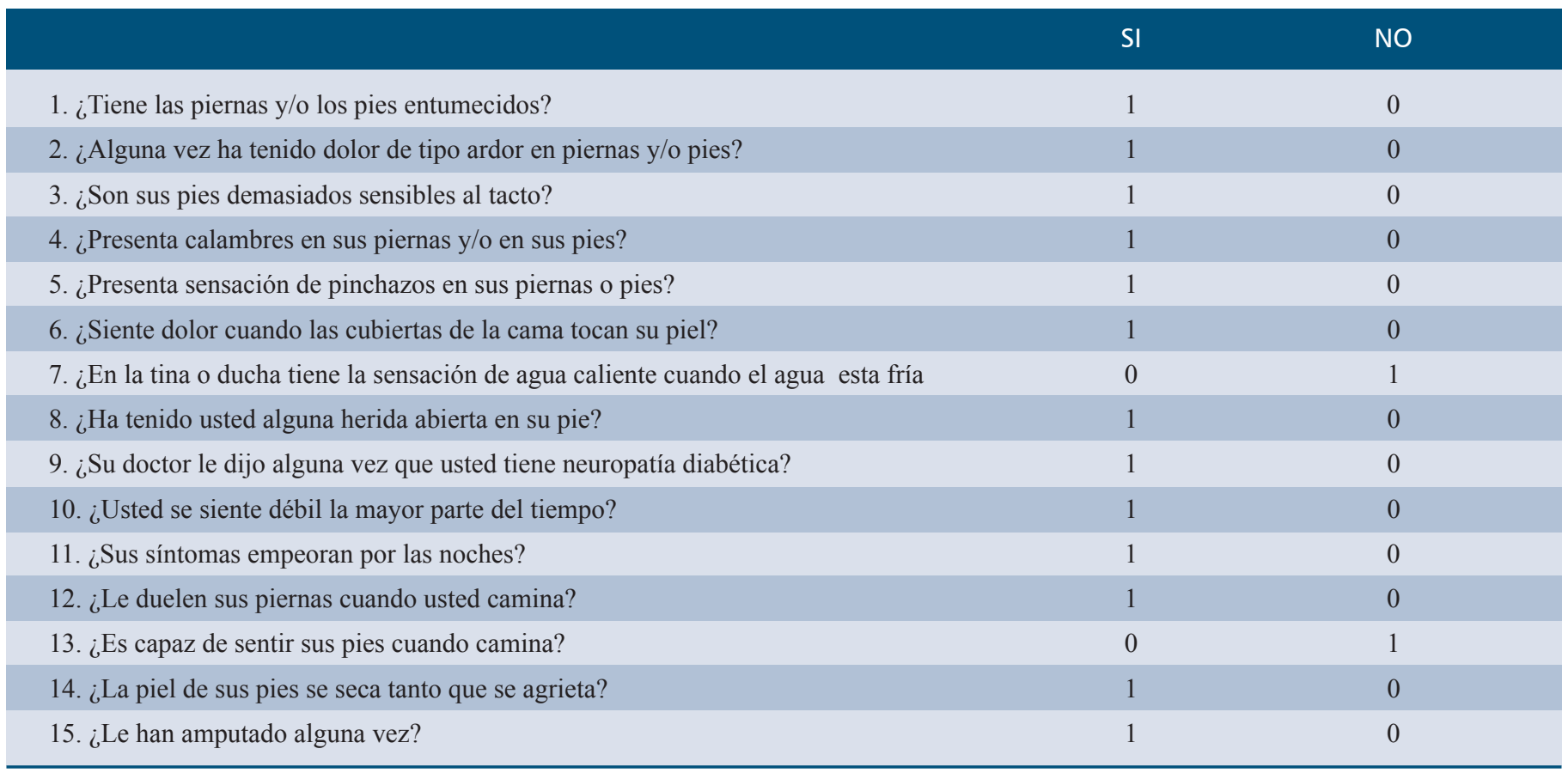

Adaptado de: Yang Z, Chen R, Zhang Y, Huang Y, Hong T, Sun F, et al. Scoring systems to screen for diabetic peripheral neuropathy. En: The Cochrane Collaboration, editor. Cochrane Database of Systematic Reviews [Internet]. Chichester, UK: John Wiley \& Sons, Ltd; 2014 [Consultado 2017 Mar 5]. Disponible en: http://doi.wiley.com/10.1002/14651858.CD010974 
sores para cada individuo (ej: calzado inadecuado, malos hábitos de higiene, etc.) y cambios en el estilo de vida de los pacientes (ej: actividad física regular, disminución en el consumo de tabaco y alcohol, entre otros) $(22,23)$.

Para el manejo del dolor neuropático existen 3 líneas de tratamiento descritas (Tabla 4). Primera línea: pueden utilizarse en monoterapia o en asocio con un fármaco tópico como la lidocaína o capsaicina en parches, la eficacia se evalúa tras 2-4 semanas del inicio, y si tras este periodo no ha resultado efectivo, se debe suspender el tratamien- to. En trabajos recientes sobre la tolerancia y eficacia de estos fármacos, la pregabalina resultó ser el fármaco más eficaz, la amitriptilina el menos seguro y la gabapentina el que mejor balance eficacia-seguridad presentaba. Se recomienda iniciar la administración de los fármacos en dosis bajas e ir titulando hasta alcanzar la dosis eficaz, si el fármaco seleccionado no funciona, es necesario cambiar a otro fármaco del mismo grupo o cambiar de grupo. Segunda línea: se recomienda comenzar con un opioide débil como tramadol y si es necesario se puede sustituir por un opioide mayor. Finalmente, los medicamentos utiliza-

TABLA 3. Examen clínico del pie (MNSI).

\section{ÍTEM}

Apariencia del pie

A. Deformidad
a. Dedos en martillo
b. Dedos sobrepuestos
c. Haluxvalgus
d. Subluxación de la articulación
e. Cabeza metatarsal prominente
f. Convexidad medial (pies de Charcot)

B. Piel seca

C. Infección o grietas

\begin{tabular}{|c|c|}
\hline Ulceración & $0=$ normal, $1=$ anormal \\
\hline Grado 1 & $\begin{array}{l}\text { Eritema no blanqueable, decoloración de la piel, edema, induración } \\
\text { o dureza. }\end{array}$ \\
\hline Grado 4 & $\begin{array}{l}\text { Destrucción extensa, necrosis del tejido o daño al músculo, el hueso } \\
\text { o tejido de sostén. }\end{array}$ \\
\hline Reflejo aquiliano & $0=$ presente, $0.5=$ presente al reforzar $1=$ ausente \\
\hline Vibración & $0=$ presente $, 0,5=$ reducido, $1=$ ausente \\
\hline Ausente & el paciente no detecta vibración \\
\hline
\end{tabular}

Adaptado de: Yang Z, Chen R, Zhang Y, Huang Y, Hong T, Sun F, et al. Scoring systems to screen for diabetic peripheral neuropathy. En: The Cochrane Collaboration, editor. Cochrane Database of Systematic Reviews [Internet]. Chichester, UK: John Wiley \& Sons, Ltd; 2014 [Consultado 2017 Mar 5]. Disponible en: http://doi.wiley.com/10.1002/14651858.CD010974 
dos en la tercera línea comprenden anticonvulsivantes, inhibidores de la recaptación de noradrenalina y dopamina y antagonistas NMDA, todos estos medicamentos deben ser administrados preferiblemente en dosis controladas, cuidando al paciente del advenimiento de efectos adver$\operatorname{sos}(24,25)$.

En la actualidad se están investigando nuevos fármacos e intervenciones que ayuden a disminuir la prevalencia de neuropatía diabética, pero el control glucémico y la educación al paciente siguen siendo las medidas más efectivas.

\section{Cardiopatía isquémica}

\section{Definición y epidemiología}

La cardiopatía isquémica (CI) se define como un desorden de la función cardiaca secundario a un flujo sanguíneo insuficiente del tejido muscular de carácter crónico dado generalmente por alteraciones ateroscleróticas estables de la pared de los vasos sub epicárdicos del corazón (26,27).

En el 2014 la OPS publicó un perfil de las enfermedades cardiovasculares (ECV) en población colombiana evidenciando una prevalencia de cardiopatía isquémica del $57 \%$ en hombres y $44 \%$ en mujeres (28). Se estima que al momento de realizar el diagnóstico inicial un 50\% presenta complicaciones vasculares previas, y al menos un $80 \%$ de las defunciones están dadas por enfermedades cardiovasculares, en mayor medida por cardiopatía isquémica y accidente vascular cerebral $(29,30)$.

\section{Fisiopatología}

Se ha visto como la DM acelera la génesis y evolución de la placa de ateroma y aumenta la concentración de especies reactivas de oxígeno (EROS) por diversos mecanismos como por ejemplo: alteración de la función mitocondrial e interacción de la glucosa con proteínas de membrana generando productos finales de glicosilación avanzada no enzimática (AGES) que no solo aumentan los radicales libres sino que se unirán a los receptores para AGE que se encuentran en diversas células como los macrófagos derivando en una activación de las vías inflamatorias. La producción de estas EROS especialmente del anión superóxido lleva a una afectación endotelial al disminuir la producción de óxido nítrico (NO). Otros mecanismos como el stress del retículo endoplásmatico y la resistencia a la insulina también disminuyen la producción de NO generando vasoconstricción.

Otras alteraciones como la apoptosis de las células del músculo liso, el aumento de la función de las metaloproteasas, el aumento de la acción de los macrófagos en la placa de ateroma, el aumento de la producción de endotelina debido a la insulinorresistencia y el aumento de la expresión de receptores de glicoproteína Ib y IIb/IIIa en las plaquetas generan vasoconstricción, promueven la inflamación, la trombosis y aceleran el proceso aterosclerótico el cual deriva en enfermedad coronaria arterial (31-34).

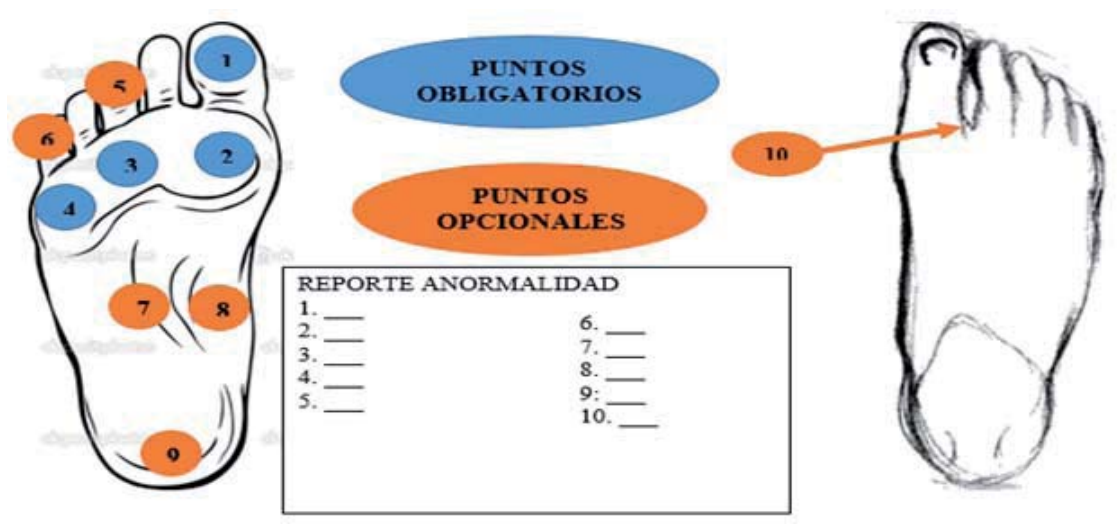

FIGURA 2. Test del monofilamento.

Adaptado de: González CP. Monofilamento de Semmes-Weinstein. Diabetes Práctica Actual Habilidades En Aten Primaria. 2010;1(1):8-19. 
TABLA 4. Principales fármacos utilizados en el Tratamiento del dolor neuropático.

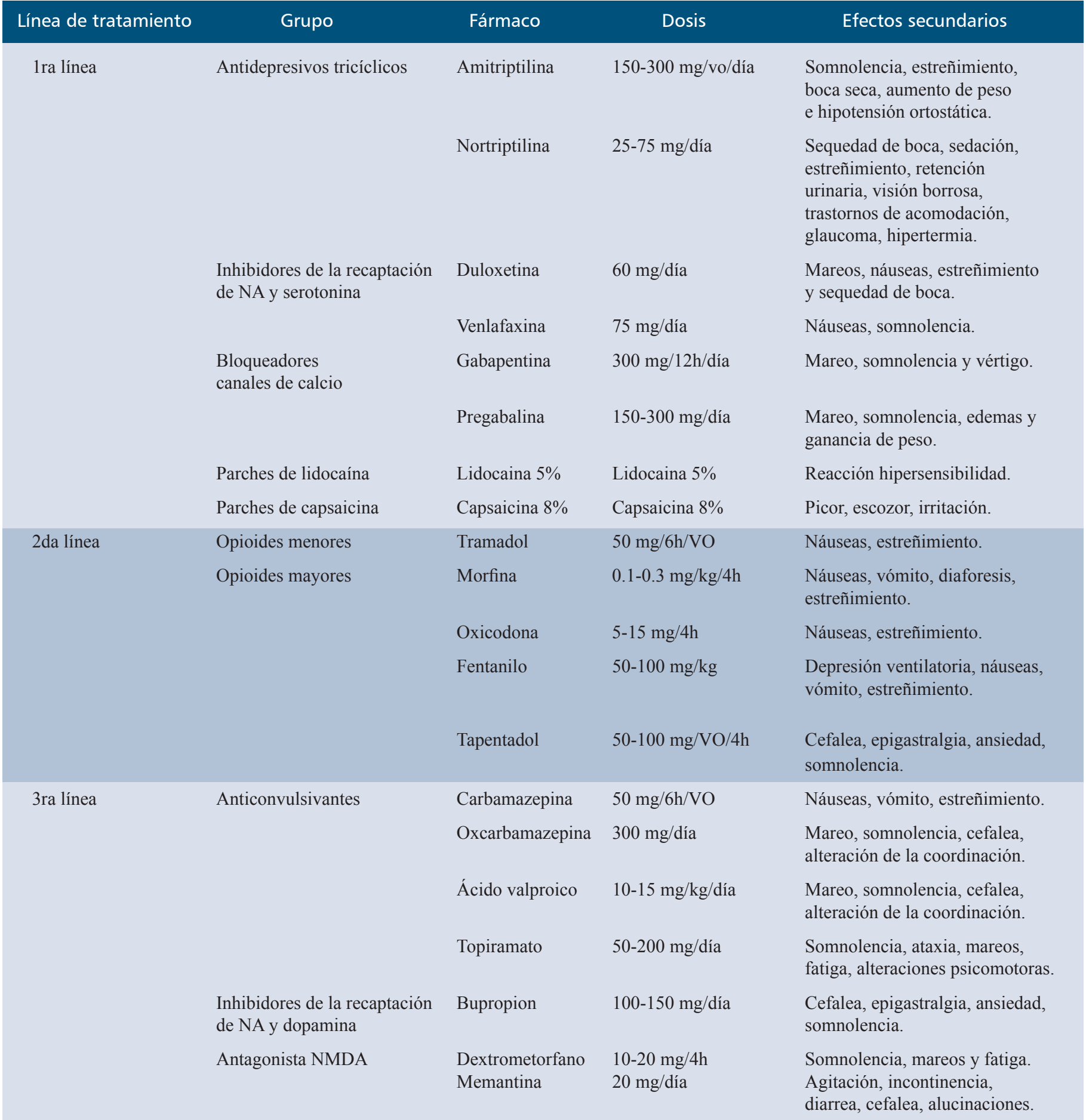

Adaptado de: Botas Velasco M, Cervell Rodríguez D, Rodríguez Montalbán AI, Vicente Jiménez S, Fernández de Valderrama Martínez I. Actualización en el diagnóstico, tratamiento y prevención de la neuropatía diabética periférica. Angiología [Internet]. mayo de 2017 [Consultado 2017 Jun 02];69(3):174-81. Disponible en: http://linkinghub.elsevier.com/retrieve/pii/S0003317016300967, San Miguel FV, Puente DM, Julià JV. Neuropatía diabética y pie diabético. Med-Programa Form Médica Contin Acreditado [Internet]. 2016 [Consultado 2017 Jun 02];12(17):971-981. Disponible en: http://www.sciencedirect.com/science/article/pii/S0304541216301457 y Dandan HR, Brunton LL. Goodman and Gilman, Manual de Farmacología y Terapéutica. 2ed. Mexico D.F: McGrawHill; 2015. 
Cuando esta enfermedad es estable, disminuye el flujo de sangre de manera crónica y se produce disminución de la presión hidrostática, disminución de los sustratos como el oxígeno y menor depuración de los productos de degradación en el tejido cardíaco generando la cardiopatía isquémica $(27,35)$.

\section{Cuadro clínico}

El cuadro de una angina típica es consistente en dolor retroesternal, opresivo, que se desencadena tras algún estrés y mejora con el reposo, la duración es menor a $20 \mathrm{~min}$ en caso de Angina Inestable (AI) y mayor de éste tiempo en caso de infarto agudo de miocardio (IAM), la intensidad es variable, puede irradiarse al brazo izquierdo, al cuello, a la mandíbula, y/o al epigastrio y estar asociado a disnea, lipotimia, náusea, diaforesis o síncope $(36,37)$.

\section{Diagnóstico}

El diagnóstico de síndrome coronario agudo (SCA) se realiza al obtener 2 de 3 criterios ya sean clínicos, electrocardiográficos o enzimáticos. El electrocardiograma (EKG) debe realizarse cada 15-30 min en caso de ser negativo; en caso de angina inestable/infarto agudo de miocardio sin elevación del segmento ST (AI/IAMSEST) puede encontrarse depresión o aplanamiento del segmento $\mathrm{ST} \geq 0.5 \mathrm{~mm}$ en dos derivaciones contiguas o inversión de la onda $\mathrm{T} \geq 1 \mathrm{~mm}$; mientras que en los pacientes con infarto agudo de miocardio con elevación del segmento ST (IAMCEST) se ve una elevación del punto $J$ en dos derivaciones contiguas $\geq 1 \mathrm{~mm}$ en todas las derivaciones excepto en V2-V3 y un segmento ST dirigido hacia arriba. En cuanto a las enzimas cardiacas, las troponinas poseen mayor sensibilidad y especificidad, se detectan luego de cuatro horas de instaurado el cuadro clínico, tienen su pico máximo desde las primeras 12 a 48 horas y disminuyen en los 5 -14 días posteriores. Permiten diferenciar entre un IAMSEST y una AI, ya que esta última no sobrepasa el percentil $99(<1 \mu \mathrm{g} / \mathrm{L})(38,39)$.

\section{Tratamiento}

El tratamiento de la cardiopatía isquémica con disfunción ventricular izquierda resulta un tema controversial. Actualmente se ha evidenciado importante mejoría de los pacientes tras revascularización miocárdica. Según la sociedad colombiana de cardiología, aproximadamente $60 \%$ de los pacientes se benefician de un aumento mínimo del $5 \%$ en la fracción de eyección del ventrículo izquierdo, en relación al porcentaje previo a la revascularización. Para brindar un pronóstico de dicha mejoría se ha postulado el uso del ecocardiograma estrés dobutamina, donde al menos el $25 \%$ del miocardio es viable. Adicional a éste método predictor se considera que pacientes con segmentos discinéticos y acinéticos, con ausencia de una cicatriz y una extensión transmural menor al $25 \%$ presentan una mejor recuperación funcional (35).

Otro método utilizado para el tratamiento de CI es el stent liberador de fármaco. El estudio aleatorizado CREDO-Kyoto encontró que la revascularización vs stent liberador de fármaco se asoció a una reducción tasa de muerte a cinco años $(\mathrm{P}=0,046)$ y dio mejores resultados en cuanto a muerte de origen cardiaca, infarto agudo al miocardio y revascularización de los vasos tratados (HR $1,45,95 \%$ CI 1,00 a $2,51, \mathrm{p}=0,047$; HR $2,31,95 \%$ CI 1,31 a $4,08, p=0,004$; y HR $3,70,95 \%$ CI 2,91 a 4,69 , $\mathrm{p}=<0,001$, respectivamente) (40). Otro estudio apoyo los resultados arrojados por CREDO-Kyoto encontrando que a cinco años la tasa de muerte fue significativamente menor con revascularización vs stent liberador de fármaco $(\mathrm{p}<0,00001)$, adicional a ello se disminuyó la tasa de infarto agudo al miocardio, la revascularización de los vasos tratados y no hubo diferencias significativas en ictus $(6,2 \%-19,2 \%$. $p<0,00001 ; 8 \%$ - 30,4 \% p $<0,00001,7,6$ $\%-6,3 \% \mathrm{p}=0,4267$ respectivamente) (41). Por el contrario, el BARI 2D evidenció una tasa de supervivencia la cual no difirió significativamente entre los pacientes con revascularización $(88,3 \%)$ y tratamiento médico $(87,8$ $\%, \mathrm{p}=0,97)$, la tasa de eventos cardiovasculares mayores en el estrato de revascularización fue significativamente menor comparado con el tratamiento médico $(30,5 \%$, $\mathrm{p}=0,01 ; \mathrm{P}=0,002$ en la interacción entre el estrato y grupo de estudio), y los eventos adversos fueron similares entre los grupos (42). En el estudio SYNTAX, sin embargo, se encontró que el stent liberador de fármaco si bien a cinco años genera mayores tasas de efectos adversos cardíacos y cerebrovasculares mayores (stent liberador de farmaco: $46,5 \%$ vs revascularización: $29,0 \%$; $\mathrm{P}<0,001$ ), en pacientes con lesiones de baja complejidad puede ser una opción terapéutica válida sin aumento en las tasas de muerte por efectos adversos (18). Cabe destacar que tanto el estudio CREDO-Kyoto como el SYNTAX evaluaban cohortes tanto de pacientes diabéticos como no diabéticos $(40,43)$. 


\section{Cardiopatía diabética}

\section{Definición}

La cardiopatía diabética (CD) se define como la afectación negativa de la función cardiaca debido a anormalidades estructurales y funcionales causadas por la Diabetes Mellitus (DM) en ausencia de enfermedades congénitas cardíacas, enfermedad arterial coronaria, hipertensión arterial (HTA) y enfermedad vascular significativa. Esta enfermedad derivará finalmente en insuficiencia cardíaca con clínica de falla con fracción de eyección reducida o preservada $(44,45)$. La CD predispone a padecer cualquier tipo de disfunción ventricular izquierda ya se diastólica, sistólica y con ello lleva a la falla cardiaca $(46,47)$.

\section{Etiopatogenia}

Las principales alteraciones metabólicas que afectan al músculo cardiaco derivadas de la diabetes se describen a continuación:

\section{Hiperlipidemia}

En condiciones normales, el corazón puede utilizar como sustrato tanto los ácidos grasos libres como la glucosa, un aumento en la expresión del cluster de diferenciación (CD36) y de la translocasa de ácidos grasos (FAT) por sobre la expresión de GLUT-4 en el sarcolema debido a la resistencia a la insulina provoca una mayor entrada de estos generando una esteatosis cardiaca. Su utilización como sustrato deriva en la activación de la kinasa piruvato deshidrogenasa 4 en la mitocondria, proceso que requiere altas cantidades de oxígeno provocando un aumento en las especies reactivas de oxígeno (EROS) y productos de degradación de lípidos los cuales generan lipotoxicidad de las células cardiacas y provocarán un desacoplamiento mitocondrial disminuyendo las reservas energéticas de la célula alterando la contracción $(48,49)$

\section{Hiperglucemia}

A mayor disposición de glucosa mayor oxidación de la misma derivando en: aumento de las EROS las cuales dañan el ADN, inhibición de la enzima glucosa 3 fosfato deshidrogenasa (G3PDH) y activación de la enzima reparadora polipolimerasa, con lo cual se producirá un desvío hacia vías de glicolisis alternas aumentando los productos finales de glicosilación avanzada (AGE) responsables en mayor medida de los daños celulares producto de la hiperglucemia, así, se produce un aumento de la producción y depósitos de colágeno en músculo cardiaco y liso arterial provocando la rigidez de los mismos, aumento de la disfunción endotelial y aterosclerosis, y junto a la mayor producción de hexosamina, el flujo de poliol, la activación de la protein kinasa c, y la expresión alterada de los canales de rianodina (RYR) y SERCA2, por el aumento de glucosa, derivan en una disfunción sistólica y diastólica tratada más adelante (48-52).

\section{Hiperinsulinemia y resistencia a la insulina}

Como se describió anteriormente, la resistencia a la insulina provocará un mayor ingreso de ácidos grasos libres al retículo sarcoplásmico, adicional a ello aumenta la degradación y disminuye la producción de óxido nítrico, aumenta el calcio intracelular y la sensibilidad al mismo generando vasoconstricción coronaria, también aumenta la fosfatasa alcalina, la expresión de osteocalcina y la activación de los receptores del NF-k $\beta$ todo ello promoviendo la rigidez vascular que derivara en el desarrollo de la enfermedad arterial coronaria (48-52).

La hiperinsulinemia a su vez causa hipertrofia cardiaca debido a: activación de la vía Pi3kalfa/ak-1, activación del sistema nervioso simpático mediante receptores beta 1 adrenérgicos, fibrosis intersticial, disminución de la contractibilidad y aumentan la apoptosis, adicionalmente aumenta la expresión de la vía MAP kinasa por encima de la IRS-1 promoviendo el crecimiento y remodelado cardíaco y promoviendo la muerte celular endotelial y miocárdica (48-52).

El aumento de EROS producirá un estrés del retículo endoplasmático que junto con el desacoplamiento mitocondrial, provocará la alteración de las proteínas transportadoras, sumado a esto, el aumento de triglicéridos intracelular altera la apertura de canales de KATP (ATPsensitive potassium channel) disminuyendo el potencial de acción, todo ello derivando en un transporte de calcio inadecuado alterando la respuesta del músculo al estímulo eléctrico, retrasando la relajación de la diástole (48-52).

También se ha encontrado una disminución del GLP-1 (glucagon like peptide-1) debido al aumento de la actividad del DPP-4 la cual disminuye la síntesis y aumenta la degradación del primero. Se han visto actividades protectoras del GLP-1 como la disminución de las cifras de 
tensión arterial y mejoría de la función cardiaca en diabéticos, proceso alterado en la DM (48-52).

El llenado normal del ventrículo izquierdo durante la diástole ocurre en un $90 \%$ de manera pasiva mientras que al final de la misma un $10 \%$ es activa. Los cambios metabólicos, estructurales y funcionales anteriormente mencionados producen una alteración de la pared muscular ventricular derivando en una discapacidad en la fase de llenado pasiva en un primer momento, la progresión de esta discapacidad generará la CD la cual se produce en tres fases a saber:

- Fase temprana: los cambios metabólicos como la resistencia a la insulina, el aumento de glucosa, la hiperlipidemia, alteran el flujo coronario. Pocos son los cambios estructurales como la rigidez cardiaca, o la alteración del llenado temprano en esta fase, la cual suplirá la contracción atrial.

- Fase avanzada: presenta cambios celulares como fibrosis, aumento de la apoptosis y necrosis, estrés oxidativo y mala respuesta inmune. Se presentan cambios estructurales como aumento de la masa ventricular, engrosamiento de la pared y un mayor tamaño ventricular izquierdo generando cambios funcionales como una marcada alteración del inicio de la diástole y una ligera disminución de la fracción de eyección.

- Fase tardía: estructuralmente hay un aumento del colágeno intersticial y perivascular, pérdida de fibras musculares, necrosis, y en los vasos coronarios pequeños hipertrofia, engrosamiento y esclerosis del músculo liso y microaneurismas capilares, que junto con las alteraciones metabólicas descritas derivaran en una activación neurohumoral las cuales generan una alteración microvascular coronaria, afectando la sístole y la diástole ventricular izquierda (48-52).

\section{Presentación clinica y diagnostico}

Actualmente se han encontrado dos patrones de presentación de la cardiomiopatía diabética, el primero hace alusión a una falla cardiaca con fracción de eyección reducida y el segundo a una falla cardiaca con fracción de eyección preservada.

\section{Falla cardiaca con fracción de eyección preservada}

En este fenotipo, el ventrículo izquierdo se encuentra de tamaño normal, rígido, con un remodelado que ocurre de forma concéntrica, con disfunción diastólica, pero sístole normal, al estudio histopatológico se encuentran cardiomiocitos hipertróficos, sarcómeras normales, alta tensión entre ellas, volumen de colágeno modificado y aumento de triglicéridos todo ello debido a la hiperglucemia, resistencia a la insulina y la lipotoxicidad. Esta es la forma de presentación de cardiopatía en pacientes que presentan diabetes tipo 2 por lo cual, al realizar el diagnóstico, el antecedente de esta enfermedad toma especial relevancia. Suele encontrarse síntomas como edema de miembros inferiores y disnea, signos como galope, S4, congestión pulmonar, distensión de las venas del cuello y hepatomegalia $(45,52)$.

La confirmación se realiza mediante estudios paraclínicos, se debe excluir la presencia de enfermedades concomitantes como enfermedad coronaria, HTA, valvulopatías y enfermedades cardiacas congénitas para lo cual la realización de una angiografía coronaria y doppler se hace necesaria. Adicional a ello se debe excluir enfermedad infiltrativa cardiaca por biopsia miocárdica $(45,52)$.

Después de descartar posibles comorbilidades, debe evaluarse la función sistólica la cual debe ser normal $(\geq 50 \%)$ con un volumen al final de la sístole $\leq 97 \mathrm{~mL} /$ $\mathrm{m} 2$ y evidencia de disfunción diastólica mediante doppler $(45,52)$.

\section{Falla cardiaca con fracción de eyección reducida}

Se ha encontrado en pacientes con diabetes tipo 1 remodelado cardiaco excéntrico, disfunción sistólica ventricular izquierda. En cuanto a los cardiomiocitos se encuentra daño en las sarcómeras con una menor contracción de las mismas, alto volumen de colágeno, muerte celular, así como depósitos de triglicéridos. Debido al daño autoinmune de la vasculatura cardiaca se evidencia alteración endotelial con reducción del flujo sanguíneo cardiaco sin presencia de enfermedad arterial coronaria $(45,52)$.

La confirmación se realiza descartando la miocarditis viral y aquellas comorbilidades anteriormente descritas, 
se debe poseer DM y pueden estar presentes síntomas como disnea y signos de congestión, S3 y galope. Se confirma el diagnóstico mediante ecocardiografía encontrándose alteración sistólica $(<50 \%)$ con volumen al final de la diástole anormal $(>97 \mathrm{ml} / \mathrm{m} 2)(45,52)$.

Como se describió anteriormente el diagnóstico de CD se da después de descartar la presencia de comorbilidades que deriven en insuficiencia cardiaca, enfermedades que se presentan frecuentemente en el paciente diabético dificultando el reconocimiento de $\mathrm{CD}$, por ende, se revisará el diagnóstico de la falla cardíaca en general, clasificación y tratamiento actual.

El diagnóstico de insuficiencia cardiaca (IC) debe estar fundamentado en una completa historia clínica y examen físico riguroso. La historia debe recabar datos como: antecedente familiar de IC, síntomas como disnea y fatiga, dolor torácico, duración, severidad, capacidad y actividad física, actividad sexual, anorexia y saciedad precoz, pérdida o ganancia de peso y palpitaciones, problemas de sueño, recientes hospitalizaciones o medicamentos para IC. Al examen físico: índice de masa corporal, presión arterial (PA) en decúbito dorsal y sentado, frecuencia de pulso y frecuencia cardíaca, ingurgitación yugular, presencia de ruidos cardiacos agregados especialmente S3 o soplos, choque de la punta, frecuencia respiratoria, estertores pulmonares o derrame pleural, hepatomegalia o ascitis, edema periférico y menor temperatura de las extremidades inferiores (53).

\section{Paraclínicos e imágenes diagnósticas}

Para el diagnóstico de falla cardiaca, diversas guías proponen el uso tanto de técnicas de imagen como de biomarcadores. Al identificar un posible caso de insuficiencia cardiaca recomiendan en primera línea el uso de ecocardiograma, química sanguínea y electrocardiograma (ECG). El uso de radiografía de tórax se discutirá más adelante $(45,52)$.

\section{Ecocardiograma}

Las guías recomiendan el uso de ecocardiograma de preferencia transtorácico de 2 dimensiones con doppler, esta técnica permite analizar el volumen de las cámaras cardíacas, la función de las válvulas, el grosor y movimiento de las paredes del corazón y la función diastólica y sistólica, esta última permite la división de la falla car- diaca en dos grandes grupos mediante la medición de la fracción de eyección pudiendo ser esta $>50 \%$ preservada o $<50 \%$ reducida. Los resultados de imagen permiten adoptar medidas terapéuticas, realizar una evaluación del estado hemodinámico y sentar un pronóstico del paciente. Las anormalidades ecocardiográficas más comúnmente halladas en falla cardiaca se exponen en la tabla $5(45,52)$.

\section{Electrocardiograma}

Su uso permite ver el estado de la conducción eléctrica del corazón e identificar el ritmo cardíaco permitiendo guiar el manejo terapéutico, adicionalmente se pueden identificar criterios de hipertrofia ventricular, fibrilación auricular, así como ondas Q las cuales pueden indicar pérdida de miocardio funcional aportando datos sobre la posible etiología de la insuficiencia cardiaca. En la tabla 6 se muestran algunos criterios electrocardiográficos para identificar bloqueo de rama izquierda, hipertrofia ventricular izquierda y fibrilación auricular. $(45,52)$.

\section{Radiografía de tórax}

No se recomienda su uso de rutina, está indicado cuando se realiza la evaluación de pacientes en quienes se sospeche una descompensación aguda de la falla cardiaca o en el debut de la enfermedad. La radiografía debe siempre correlacionarse con los hallazgos clínicos del paciente, se deben buscar signos de edema intersticial o alveolar, analizar el tamaño de la silueta cardiaca, y analizar otras posibles enfermedades torácicas que puedan ser causantes de la sintomatología encontrada (53-55).

\section{Química sanguínea}

La sociedad europea de cardiología recomienda la medición del péptido natriurético tipo $\mathrm{A}$ o $\mathrm{B}$ y propone diversos puntos de corte para ambos, adicional a ello, sodio, potasio, calcio, urea, enzimas hepáticas, hormonas tiroideas, creatinina, estimación de la tasa de filtración glomerular y la realización de un hemograma, todo ello con el fin de analizar el mejor manejo terapéutico, realizar un adecuado seguimiento, detectar causas de la fallas cardiaca y obtener un pronóstico de la enfermedad (54).

\section{Clasificación}

La American Heart Association (AHA) usa la evaluación de las alteraciones estructurales y los factores de riesgo para el desarrollo de IC como medio para clasificar 
en cuatro estadios a los pacientes, con lo cual arroja información sobre el desarrollo y progresión de la enfermedad (tabla 7). Así, una progresión en la clasificación se asocia a una reducción del 5\% en la sobrevida de estos pacientes, la estratificación permite enfocar los esfuerzos terapéuticos a cada estadio en particular (53).

TABLA 5. Anormalidades ecocardiográficas más comúnmente halladas en falla cardiaca.

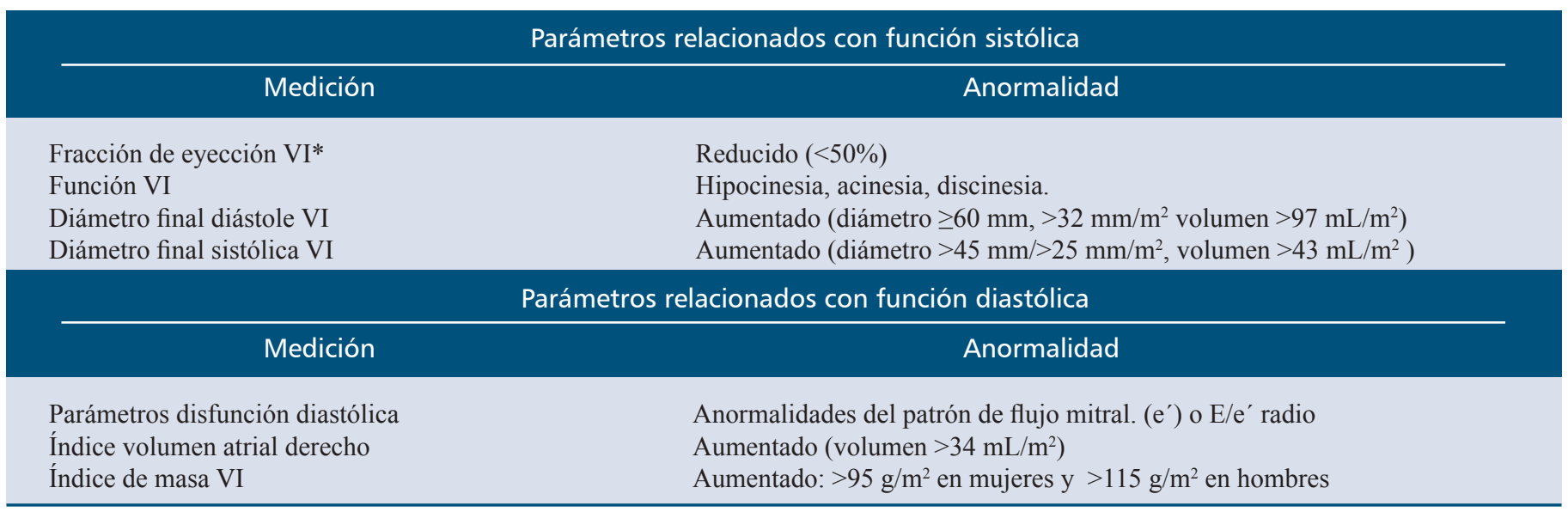

*Ventrículo izquierdo. Adaptado de: McMurray J, Adamopoulos S, Anker S, Auricchio A, Bohm M, Dickstein K et al. ESC Guidelines for the diagnosis and treatment of acute and chronic heart failure 2012: The Task Force for the Diagnosis and Treatment of Acute and Chronic Heart Failure 2012 of the European Society of Cardiology. Developed in collaboration with the Heart Failure Association (HFA) of the ESC. European Heart Journal. 2012;33(14):1787-1847.

TABLA 6. Criterios electrocardiográficos para identificación de bloqueo de rama izquierda, hipertrofia ventricular izquierda y fibrilación auricular.

\begin{tabular}{|c|c|}
\hline Hallazgos & Criterios \\
\hline Hipertrofia ventricular izquierda & $\begin{array}{l}\text { a. Índice de Sokolow-Lyon: } \\
-\mathrm{SV} 1+\mathrm{R} \text { V5 o v6: }>3,5 \mathrm{mV} \text { o } \mathrm{S} \text { de } \mathrm{DI}+\mathrm{R} \text { de } \mathrm{V} 6:>3,5 \mathrm{mV} \\
-\mathrm{R} \mathrm{aVL}>1,1 \mathrm{mV} \\
\text { b. Medición del producto voltaje-duración de Cornell: } \\
\text { - Duración de QRS } \times \text { voltaje de Cornell } \\
>2.436 \mathrm{~mm}-\mathrm{s} \text { ( } \mathrm{seg} \times \mathrm{mv}) \\
\text {-Duración de QRS } \times \text { suma de voltajes en todas } \\
\text { las derivaciones }>1.742 \mathrm{~mm}-\mathrm{s}(\mathrm{seg} \times \mathrm{mv}) \\
\text { (Criterios de voltaje de Cornell: } \mathrm{S} \text { en } \mathrm{V} 3+\mathrm{R} \text { en aVL } \geq 2,8 \mathrm{mV} \text { (hombres); } \\
\mathrm{SV} 3+\mathrm{RaVL}>2 \mathrm{mV} \text { (mujeres)) } \\
\text { c.Onda } \mathrm{S} \text { más profunda }+ \text { onda } \mathrm{S} \text { de } \mathrm{V} 4(\geq 2.3 \mathrm{mV} \text { mujeres } \mathrm{y} \geq 2.8 \mathrm{mV} \text { hombres) }\end{array}$ \\
\hline
\end{tabular}

Adaptado de: Abedin Z y Conner R. ECG Interpretation, The Self-Assesment Approach. 2ed. Texas. Blackwell Futura; 2008. 
La clasificación de la New York Heart Association (NYHA) evalúa la sintomatología de los pacientes y su capacidad física ubicándolos en uno de los cuatro grupos, si bien esta clasificación depende en parte del juicio del médico examinador y cambia en periodos de tiempo permite predecir la mortalidad de los pacientes con IC (tabla 8) (53).

Se ha realizado una clasificación alterna en búsqueda de una mejor evaluación de los pacientes con IC con el objetivo de establecer un pronóstico más preciso y ofrecer un mejor enfoque terapéutico (56). La clasificación HLM (tabla 5) propone la evaluación de parámetros cardíacos $(\mathrm{H})$, pulmonares (L) y la función de otros órganos (M). A nivel cardiaco tiene en cuenta: hipertrofia ventricular, infarto previo con o sin elevación del segmento ST, remodelado ventricular, fracción de eyección $<35 \%$ o flujo transmitral para dar una estratificación de 1-4. En cuanto a los parámetros de alteraciones pulmonares se evalúa si hay hipertensión pulmonar precapilar $(<15 \mathrm{mmHg}) \mathrm{o}$ poscapilar $(>15 \mathrm{mmHg})$, derrame pleural o edema pulmonar dando como resultado tres estadios de daño pulmonar. Por último, analiza diversos parámetros de daño renal, hepático, sistema nervioso central dando 4 estadios. Esta clasificación busca correlacionar los diferentes daños en estos órganos causados tanto por la IC como por las co- morbilidades y factores de riesgo que suelen encontrarse en estos pacientes (tabla 9). Sin embargo, son necesarios más datos clínicos y nuevos estudios para su validación (57).

\section{Tratamiento}

El tratamiento del paciente con IC debe estar enfocado en la estabilización de la enfermedad disminuyendo los signos y síntomas como la disnea y el edema, disminuir la probabilidad de hospitalizaciones; mejorar la sobrevida y calidad de vida de los pacientes. La sociedad europea de cardiología recomienda primero clasificar al paciente en función de su fracción de eyección, bien sea preservada o reducida permitiendo dar un tratamiento diferente a cada grupo, a su vez, dividen las recomendaciones de cada grupo de acuerdo a la clasificación de la NYHA dando patrones de manejo desde NYHA II. Para pacientes con fracción de eyección reducida el manejo debe comenzar con el uso de diuréticos para aliviar los signos y síntomas de congestión, seguido de la adición de inhibidores de la enzima convertidora de angiotensina (IECA) o antagonistas de los receptores de angiotensina (ARA II) en caso de no ser tolerados los primeros. Adicionar betabloqueadores y hacer seguimiento buscando mejoría de la sintomatología. El uso de antagonistas de la aldosterona como

TABLA 7. Clasificación ACCF/AHA.

A Con alto riesgo para IC* pero sin alteraciones cardiacas estructurales o síntomas de IC.

B Alteraciones cardiacas estructurales sin signos o síntomas de IC.

C Alteraciones cardiacas estructurales con sintomatología previa o actual de IC.

D IC refractaria la cual requiere intervenciones especializadas.

*IC: Insuficiencia cardiaca. Adaptado de: Low Wang C, Hess C, Hiatt W, Goldfine A. Clinical Update: Cardiovascular Disease in Diabetes Mellitus. Circulation. 2016;133(24):2459-2502.

TABLA 8. Clasificación funcional NYHA.

I Sin limitación de la actividad física.

II Leve limitación de la actividad física. Sin síntomas en reposo pero actividad física ordinaria causa sintomatología de IC*.

III Marcada limitación de la actividad física. Sin síntomas en reposo pero menos de la actividad física ordinaria causa sintomatología de IC.

IV Incapaz de realizar actividad física sin síntomas de IC o síntomas de IC en reposo

*IC: Insuficiencia cardiaca. Adaptado de: Low Wang C, Hess C, Hiatt W, Goldfine A. Clinical Update: Cardiovascular Disease in Diabetes Mellitus. Circulation. 2016;133(24):2459-2502. 
TABLA 9. Clasificación HLM.

Corazón (H)

Pulmones (L)

L-0 Sin compromiso

L-1 Congestión hemodinámica

L-2 Congestión clínica

L-3 Pulmón cardiaco*
H-1 Disfunción sistólica o diastólica alterada sin daño estructural

H-2 Disfunción sistólica o diastólica y alteración estructural (hipertrofia, infarto previo)

H-3 Disfunción sistólica y diastólica con remodelado ventricular izquierdo

H-4 disfunción sistólica y diastólica biventricular
M-3 Daño múltiples órganos

Disfunción otros órganos (M)

M-0 Sin alteración

M-1 Alteración de un órgano debido a IC

M-2 Alteración 2 órganos debido a IC

*Arterialización de la vasculatura precapilar y poscapilar pulmonar. IC: Insuficiencia cardiaca. Modificado de Fedele et al. Heart Failure. J Am Coll Cardiol. 2014 May 20;63(19):1959-60.

digoxin o hidralazina debe ser tomado en cuenta si el paciente continúa en clasificación NYHA II-IV y hay presencia de bloqueo de rama izquierda, taquicardia sinusal, prolongación del QT entre otros signos $(53,54)$.

En cuanto al manejo farmacológico de los pacientes con falla cardiaca con fracción de eyección preservada no hay suficientes datos significativos sobre el uso de medicamentos que reduzcan la morbilidad y mortalidad. Algunos diuréticos junto con los ARA II y los IECA pueden ser usados, adicional a ello, anticálcicos como el verapamilo pueden mejorar la capacidad física de estos pacientes. En cuanto a la guía de la $\mathrm{AHA} / \mathrm{ACCF}$ las recomendaciones están dadas para los diversos niveles en que se estratifique al paciente pudiendo ser $\mathrm{A}, \mathrm{B}, \mathrm{C}$ o $\mathrm{D}$, adicionalmente es usada la clasificación de la NYHA correlacionando esta con los estadios $\mathrm{C}$ y D para dar las recomendaciones. Para pacientes en estadio A deben controlarse los factores de riesgo que puedan derivar en falla cardiaca, para el estadio B debe considerarse el uso de ARA II, IECA o betabloqueadores bajo ciertas circunstancias como infarto previo, fracción de eyección reducida asintomática entre otras situaciones junto con cambios en el estilo de vida $(53,54)$.

En los estadios C y D además de los cambios en el estilo de vida, se recomienda el uso de tratamiento farmacológico el cual se debe escoger procurando evitar la mayor cantidad de efectos adversos para el paciente pero que garanticen un adecuado control de la enfermedad. Para el estadio $\mathrm{C}$ con fracción de eyección preservada el uso de diuréticos está indicado junto con el tratamiento de las comorbilidades que impidan el control de los sín- tomas. Para el mismo estadio en paciente con falla cardiaca con fracción de eyección reducida están indicados el uso de diuréticos, IECA, ARA II y antagonistas de la aldosterona. Previa individualización del paciente otros medicamentos deben usarse de acuerdo a la evolución de la enfermedad. En el estadio D refractario al tratamiento está indicado el uso de terapias avanzadas, consideración de trasplante cardiaco, uso de inotrópicos crónicos positivos entre otros. $(53,54)$.

\section{Nefropatía diabética}

\section{Definición}

Se define como nefropatía diabética (ND) aquellas lesiones renales originadas por afección microangiopática, es decir es una complicación vascular crónica, exclusiva de la DM, en la que se afecta la microcirculación renal la cual genera tanto alteraciones funcionales como estructurales encontrándose principalmente una afección a nivel glomerular. La ND consiste en la presencia de albuminuria persistente (igual o superior a $300 \mathrm{mg} / 24 \mathrm{~h}$ o igual o superior a $200 \mathrm{mg} / \mathrm{min})$. Esta definición es válida tanto para la DM tipo 1 (DM1) como para la DM tipo 2 (DM2). La presencia de microalbuminuria, es decir, excreción urinaria de albúmina entre 30 y $300 \mathrm{mg} / 24 \mathrm{~h}$ ) se define como nefropatía incipiente (58).

\section{Epidemiología}

En el mundo industrializado, la DM es la primera causa de enfermedad renal terminal (ERT). A pesar de los avances en la atención a los pacientes con DM, tanto la incidencia como la prevalencia de ERT secundaria a DM 
siguen aumentando. En EE.UU. más del 30\% de los enfermos que requieren diálisis o trasplante renal tienen ERT a consecuencia de la ND y el $40 \%$ de los nuevos casos de ERT son atribuibles a la DM. En la actualidad, más de 200.000 pacientes están siendo tratados por ERT secundaria a DM (59).

La ERC afecta del 10\% al 16\% de adultos, constituyendo un serio problema mundial (60). En Suramérica, la prevalencia de DM y de ERC en estadios terminales (ERCT) incrementó en las últimas décadas, existiendo gran disparidad entre los países respecto al acceso a diálisis $(61,62)$.

En Ecuador, la prevalencia de pacientes que recibieron tratamiento sustitutivo de la función renal fue de 406 personas por millón de habitantes, en el 2010 (2). Por otra parte, los inhibidores del sistema renina-angiotensina-aldosterona (SRA) constituyen la mejor opción terapéutica para la ND, pero el riesgo residual de ERCT permanece elevado y la asociación de estas drogas estuvo relacionada con hiperpotasemia e insuficiencia renal aguda (IRA) $(63,64)$.
La prevalencia e incidencia de ERC en Colombia no se conoce con exactitud, pero se calcula que ha tenido un aumento progresivo, debido a su asociación con múltiples factores de riesgo (65). En el caso colombiano, el 28\% de la población diabética y entre el 21 y el $36 \%$ de la población hipertensa desarrollan ERC (66).

Desde la perspectiva de la ERCT, un análisis de sobrevida entre pacientes que ingresaron al programa de diálisis peritoneal y hemodiálisis entre 2001 y 2003, donde no solo se reconoce la DM como precursora de ERC (35.9\% de los casos que ingresaron a hemodiálisis y $45.3 \%$ de los casos que ingresaron a diálisis peritoneal), sino además como condicionante de sobrevida una vez instaurada la nefropatía. En el análisis por intención de tratar (a partir de un modelo de Cox univariado) el riesgo de no sobrevida se asoció con la presencia de DM con un Hazard Ration (HR) de 2.34 [IC 95\% (1.8 - 2.9)], al igual que en el análisis de casos tratados, donde la diabetes tuvo un HR de 2.19 [IC 95\% (1.77 - 2.73)]; en los análisis multivariados se ratificó la asociación entre la presencia de DM y el mayor riesgo de no sobrevida en ERC (67).

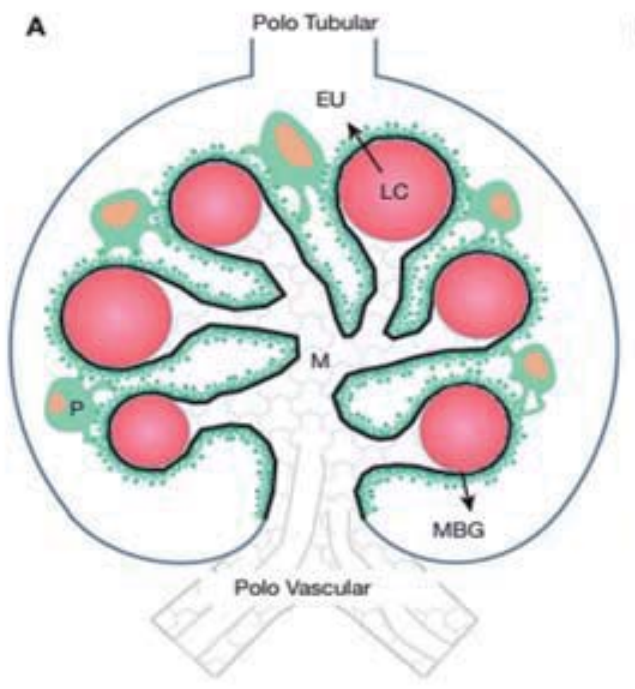

B

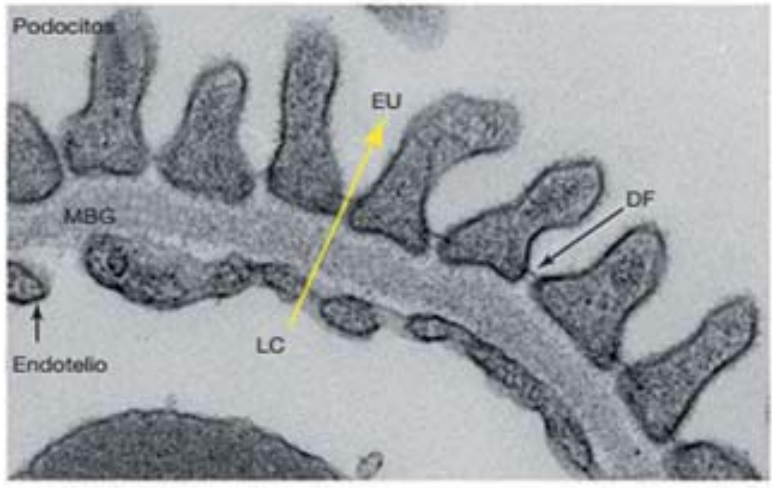

FIGURA 3. Barrera de filtración glomerular. A: representación esquemática del glomérulo, la barrera filtración glomerular (BFG) formada por los podocitos (P), la membrana basal glomerular (MBG) y el endotelio. El ultrafiltrado del plasma atraviesa la BFG (flecha negra) para llegar al espacio urinario (EU). Los podocitos (verde) contactan varios capilares glomerulares (representados como círculos rojos) y el mesangio intraglomerular (M). La MBG (línea negra) envuelve los capilares y rodea al mesangio. El endotelio glomerular está simbolizado por una línea celeste entrecortada, ubicada entre la luz capilar (LC) y la MBG, el polo vascular en la parte inferior del glomérulo, el polo tubular en la parte superior. B: Ultraestructura de la BFG observada con un microscopio electrónico. Los podocitos, la MBG, el diafragma de filtración (DF) y el endotelio fenestrado. El ultrafiltrado del plasma atraviesa la BFG, flecha amarilla, desde la luz capilar (LC) al espacio urinario (EU). Adaptado de: Carranza K, Veron D, Cercado A, Bautista N, Pozo W, Tufro A, et al. Aspectos celulares y moleculares de la nefropatía diabética, rol del VEGF-A. Nefrología. 2015;35(2):131-138. 


\section{Etiopatogenia}

La ND se acompaña muy frecuentemente de HTA y de disminución de la función renal. Es por esta razón que es sumamente importante realizar una detección precoz de los pacientes con ERC y su tratamiento es fundamental para disminuir la morbilidad cardiovascular y la velocidad de progresión de la enfermedad renal, reduciendo los costes para el sistema sanitario $(68,69)$.

Parece haber diferentes procesos patogénicos que conducen a la ND. La glomeruloesclerosis, por ejemplo, puede ser el resultado de la hipertensión intraglomerular inducida por la vasodilatación renal o de la lesión isquémica inducida por la arterioesclerosis hialina de los vasos que irrigan los glomérulos (70).

La hiperglucemia es responsable de la glucosilación no enzimática de proteínas circulantes y estructurales. Este proceso es capaz de inducir hiperfiltración glomerular e incrementar la secreción de citocinas y factores de crecimiento que producen proliferación mesangial e incremento de la matriz extracelular. Junto con el aumento en la expresión y síntesis de TGF- $\beta$ y PDGF, la hiperglucemia por sí misma aumenta la expresión de receptores AT 1 de angiotensina II que se encargan de la vasoconstricción, la proliferación vascular y la inflamación principalmente llevando a un cúmulo de matriz extracelular por aumento de producción y reducción de la degradación del colágeno (70). La activación de citocinas, elementos profibroticos, inflamación, y factores de crecimiento vascular (factor de crecimiento endotelial vascular, VEGF) pueden estar involucrados en la acumulación de la matriz en la ND (71).

El sistema renina-angiotensina (SRA) juega un papel importante en el control de la PA sistémica, la presión intraglomerular, la proteinuria y la infiltración de macrófagos que, entre otros, perpetúan la destrucción del tejido renal el cual, en su afán de restituir su morfología y función, favorece los procesos de fibrosis glomerular. La proteinuria supone un fracaso de la barrera de filtración glomerular, esta barrera limita el paso de macromoléculas en función de su tamaño, su forma y sus cargas eléctricas ya que aquellas con carga negativa no pueden atravesar con facilidad. La barrera de filtración glomerular está constituida por una capa de células endoteliales, una membrana basal y células especializadas llamadas Podocitos (Figura 3) (72).
En la nefropatía se desarrolla un estado de hiperfiltración glomerular, seguido de esto se produce un aumento de la presión capilar glomerular la cual es sostenida y daña directamente los capilares glomerulares y distiende las células mesangiales, lo que resulta en aumento de la síntesis de citocinas con capacidad de inducir proliferación y fibrogénesis. A nivel molecular en la ND sobresalen una serie de cambios importantes como el incremento de la expresión de renina y de angiotensinógeno en las células mesangiales que junto a los podocitos sintetizan angiotensina II y expresan receptores de angiotensina (72).

El aumento de angiotensina II estimula la expresión de TGF-Beta, VEGF-A, factor de crecimiento del tejido conectivo (CTGF), interleukina 6 y proteína quimiotáctica para monocitos-1 induciendo expansión de la membrana extracelular y apoptosis de los podocitos. Desde el punto de vista histopatológico, estos cambios se traducen en hipertrofia glomerular y, finalmente, glomerulosclerosis (73).

\section{Cuadro clínico}

En la ND es importante identificar la fase preclínica la cual consiste en una etapa de normo o micro albuminuria y una fase clínica caracterizada por macro albuminuria documentada, también pueden verse, aunque muy poco frecuente, casos de hematuria. El sedimento de la orina en la ND es generalmente leve, pero se ve una hematuria microscópica que puede ocurrir en cualquier forma de enfermedad glomerular, incluyendo trastornos tales como nefropatía membranosa que no está asociado con glomerulonefritis. En esta progresión de la enfermedad el aumento de la presión capilar parece ser un factor decisivo de la progresión de la ND, en esta fase la PA es aún normal. La retención de líquidos se observa tempranamente en el curso de la enfermedad renal, es decir, en una etapa caracterizada por la función renal bien conservada y sólo una modesta reducción en el nivel sérico de albúmina. Cuando se desarrolla micro albuminuria persistente de al menos $30 \mathrm{mg} / 24 \mathrm{hr}$ y después de aproximadamente 10 años de evolución de la enfermedad, la filtración glomerular puede mantenerse normal o elevarse debido a los cambios histológicos renales como el engrosamiento de la membrana basal glomerular y tubular. La micro albuminuria es más específica de la ND por DM1 que por DM2 
debido a que en estos pacientes es mayor la prevalencia de HTA que por sí misma puede producir micro albuminuria. Imanishi y colegas han demostrado que la hipertensión glomerular está presente en pacientes con DM2 y nefropatía temprana y que ésta se encuentra estrechamente correlacionada con el aumento de excreción de albúmina urinaria. La albuminuria y el edema periférico son a menudo los primeros signos de ND (75).

Cuando el aumento severo de albuminuria, llamado anteriormente "macroalbuminuria" (definido como la excreción urinaria de albúmina superior a $200 \mathrm{mg}$ /día o por encima de $300 \mathrm{mg} / \mathrm{g}$ de creatinina) precede al desarrollo de albuminuria grave, se considera como un hallazgo de mal pronóstico, en esta fase se suele presentar una proteinuria franca (más de $500 \mathrm{mg}$ de proteínas totales/día) detectable con una tira para proteínas urinarias. En esta fase la filtración glomerular (FG) suele estar por debajo de las cifras normales, la hipertensión suele hacerse más difícil de controlar y la biopsia renal muestra la lesión de Kimmelsteil-Wilson patognomónica de la ND avanzada (presente en el 25\% de los pacientes) otro rasgo patognomónico es la hialinosis de las arteriolas aferentes y eferentes que puede distinguirse de la lesión arteriolar de la hipertensión esencial (solo afecta la eferente) (76).

En la ND franca, hay un declive de la función renal y la FG comienza a ser cada vez menor, aunque puede mantener la creatinina sérica en cifras normales. En las fases más avanzadas hay una insuficiencia renal crónica con cifras de proteinuria correspondiente a síndrome nefrótico (76).

\section{Diagnóstico}

Dentro de los fundamentos para el diagnóstico de la ND se debe tener en cuenta que del 20 a 30\% de los diabéticos tienen microalbuminuria casi 15 años después de que se diagnostica DM por primera vez. Inicialmente se incrementa la tasa de filtración glomerular (GFR por sus siglas en ingles), posteriormente regresa a la normalidad a medida que se produce más daño renal, para finalmente continuar disminuyendo llegando a tener una proteinuria $>1 \mathrm{~g} / \mathrm{m} 2 / \mathrm{d}$, es decir, en rango nefrótico. En el ultrasonido pueden apreciarse riñones de características normales o aumentadas de tamaño; la biopsia muestra expansión de la matriz mesangial, glomeruloesclerosis difusa e intercapilar nodular, esta última es patognomónica de la ND (77).
Al principio, la ND se manifiesta por proteinuria; más tarde, conforme la función renal se deteriora, se acumulan urea y creatinina en la sangre. Los métodos sensibles de radioinmunoanálisis para detectar pequeñas cantidades de albúmina urinaria permiten la detección de concentraciones en microgramos, a diferencia de las tiras reactivas menos sensibles. Las recolecciones convencionales de orina de 24 horas, además de causar molestias al paciente, también muestran una amplia variabilidad en la excreción de albúmina, porque varios factores, como la postura erguida sostenida, las proteínas dietéticas y el ejercicio, tienden a incrementar las tasas de eliminación de albúmina. Por tales razones, se prefiere el cálculo del índice albúminacreatinina en una muestra urinaria matutina temprano al despertar. En la orina matutina temprana, se considera normal un índice de albúmina $(\mu \mathrm{g} / \mathrm{L}) /$ creatinina $(\mathrm{mg} / \mathrm{L})$ menor de $30 \mu \mathrm{g} / \mathrm{mg}$ de creatinina y un índice de 30 a 300 $\mu \mathrm{g} / \mathrm{mg}$ de creatinina sugiere microalbuminuria anormal. Se necesita que al menos dos muestras matutinas de orina sean anormales en un periodo de tres a seis meses para determinar el diagnóstico de microalbuminuria. La hiperglucemia a corto plazo, el ejercicio, infecciones de vías urinarias, insuficiencia cardiaca y la enfermedad febril aguda pueden ocasionar albuminuria transitoria y por ello se aconseja posponer la valoración de microalbuminuria hasta la resolución de estos problemas (77).

Por último, la disfunción avanzada asociada a hipervolemia, HTA, acidosis metabólica, hiperpotasemia, hiperfosfatemia, hipocalcemia, anemia y trastornos minerales conllevan a ERCT. En los adultos mayores de 40 años la tasa de progresión renal normal es de 0,7-1 ml/min/1,73 $\mathrm{m} 2$ año (78). Se puede considerar que un paciente presenta progresión renal anormal cuando el descenso de la FG es $>5 \mathrm{ml} / \mathrm{min} /$ año o $>10 \mathrm{ml} / \mathrm{min}$ en cinco años (79) La evaluación de la progresión de la ERC se basa en dos aspectos fundamentales (Tabla 10), el primero es la progresión a una categoría superior o más grave de deterioro en la función renal (estadio 1-5) o de albuminuria $(<30$, $30-299,>300 \mathrm{mg} / \mathrm{g}$ creatinina) y el segundo es el porcentaje de cambio respecto a la situación basal $(>25 \%$ de deterioro en el FG) o más del $50 \%$ de incremento en el cociente albúmina/creatinina. Para la valoración de la progresión renal se recomienda la estimación de la FG basal y la albuminuria, así como identificar aquellos factores de progresión renal (Tabla 11) (80); para realizar este procedimiento las nuevas guías recomiendan el cambio de la ecuación para estimar el FG a la fórmula CKD-EPI (CKD Epidemiology Collaboration) de 2009 (81). 
TABLA 10. Clasificación de la ERC por grupos según FG y pronóstico de ERC por filtrado glomerular estimado y albuminuria

Pronóstico de la enfermedad renal crónica y

albuminuria: KDIGO 2012.
Categorías por albuminuria

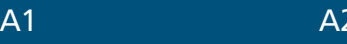

A3

Normal o levemente Aumento moderado Aumentado grave

aumentado

$<30 \mathrm{mg} / \mathrm{g}$

$<3 \mathrm{mg} / \mathrm{mmol}$

$30-299 \mathrm{mg} / \mathrm{g}$

$3-29 \mathrm{mg} / \mathrm{mmol}$

$\geq 300 \mathrm{mg} / \mathrm{g}$

$\geq 30 \mathrm{mg} / \mathrm{mmol}$
Categorías por $\mathrm{FGe}$ $\left(\mathrm{ml} / \mathrm{min} / 1,73 \mathrm{~m}^{2}\right)$

\begin{tabular}{lll} 
G1 & Normal o alto & $>90$ \\
\hline G2 & $\begin{array}{l}\text { Levemente } \\
\text { disminuido }\end{array}$ & $60-89$ \\
\hline G3a & $\begin{array}{l}\text { Descenso } \\
\text { leve-moderado }\end{array}$ & $45-59$ \\
\hline G3b & $\begin{array}{l}\text { Descenso } \\
\text { moderado-grave }\end{array}$ & $30-44$ \\
\hline G4 & Descenso grave & $15-29$ \\
\hline G5 & Fallo renal & $<15$ \\
\hline
\end{tabular}

ERC: enfermedad renal crónica; FGe: filtrado glomerular estimado; KDIGO: Kidney Disease Improving Global Outcomes.

Nota: Los colores mostrarían el riesgo relativo ajustado para cinco eventos (mortalidad global, mortalidad cardiovascular, fracaso renal tratado con diálisis o trasplante, fracaso renal agudo y progresión de la enfermedad renal) a partir de un metaanálisis de cohortes de población general. El riesgo menor corresponde al color verde (categoría «bajo riesgo»; si no hay datos de lesión renal, no se puede catalogar siquiera como ERC), seguido del color amarillo (riesgo «moderadamente aumentado»), naranja («alto riesgo») y rojo («muy alto riesgo»), que expresan riesgos crecientes para los eventos mencionados. Reproducido con permiso de KDIGO (78).

Cociente albúmina/creatinina: $1 \mathrm{mg} / \mathrm{g}=0,113 \mathrm{mg} / \mathrm{mmol} .30 \mathrm{mg} / \mathrm{g}$ $(3,4 \mathrm{mg} / \mathrm{mmol})$.

Como puede apreciarse, los estadios según el FG se denominan ahora G1 a G5 y se confirma la división del estadio 3 en dos subgrupos: G3a y G3b, división también útil para determinar la prioridad de la derivación y las diferencias de riesgo
Control por nefrología.

Control por Atención Primaria u otras especialidades.

Nota: Los números en las casillas son las visitas anuales

Adaptado de: Improving Global Outcomes (KDIGO) CKD Work Group. KDIGO Clinical Practice Guideline for the Evaluation and Management of Chronic Kidney Disease. Kidney Int Suppl 2013; 3:S6-308.

\section{Tratamiento}

Los principales objetivos del tratamiento son el control de la glucemia, de la HTA, de los lípidos y la restricción de proteínas. Como demuestran los estudios DCCT15 para la DM1 y UKPDS16 y Kumamoto Study
26 para la DM2, el control estricto de la glucemia puede retrasar o prevenir la aparición de microalbuminuria, el desarrollo de la ND, Reduce el paso de normoalbuminuria a micro o macroalbuminuria, tanto en DM1 como en DM2 (81). Por el contrario, sus efectos en la regresión de la microalbuminuria o de la nefropatía clínica han sido 
TABLA 11. Factores relacionados con progresión renal anormal.

Caso 1. Paciente masculino de 55 años de edad, raza negra, creatinina: 2 (mg/dL), proteínas en parcial de orina: 280 (mg/g). Resultado por fórmula CKD-EPI: $42,3(\mathrm{~mL} / \mathrm{min} / 1,73 \mathrm{~m} 2)$ y albuminuria en A2. Paciente en estadio renal G3b (Descenso moderado-grave) y A2 con aumento moderado de la albuminuria. Este paciente debe tener control por Atención Primaria u otras especialidades.

Caso 2. Paciente femenino de 50 años de edad, raza blanca, creatinina: 2,3 (mg/dL), proteínas en parcial de orina: 350 (mg/g). Resultado por fórmula CKD-EPI: $24(\mathrm{~mL} / \mathrm{min} / 1,73 \mathrm{~m} 2)$ y albuminuria en A3. Paciente en estadio renal G4 (Descenso grave) y A3 con aumento grave de la albuminuria. Este paciente debe estar en control por nefrología 4 veces o más por año.

Adaptado de: Martínez-Castelao A, Górriz JL, Bover J, Segura-de la Morena J, Cebollada J, Escalada J, et al. Documento de consenso para la detección y manejo de la enfermedad renal crónica. Aten Primaria. 2014;46(9):501-519 e Improving Global Outcomes (KDIGO) CKD Work Group. KDIGO Clinical Practice Guideline for the Evaluation and Management of Chronic Kidney Disease. Kidney Int Suppl 2013; 3:S6-308.

menos evidentes. Además, recientemente se ha observado que el control demasiado estricto de la glucemia puede tener complicaciones cuando se produce hipoglucemia. En cuanto al uso de hipoglucemiantes orales en la insuficiencia renal en estadio III a V, las sulfonilureas y las biguanidas deben ser evitadas, mientras que están aceptados las glinidas y los inhibidores de la DPP-4 (82).

El control de la PA es un pilar fundamental del tratamiento de la ND. Como prevención primaria, en la fase de normoalbuminuria, la reducción de la PA, especialmente con el bloqueo del sistema renina-angiotensina con IECA o ARA-II, retrasa la aparición de la microalbuminuria en la DM1 y la DM2. En la fase de microalbuminuria el bloqueo del sistema renina-angiotensina mediante IECA en la DM1 o de ARA-II en la DM2 es la medida más eficaz para frenar la progresión desde microalbuminuria a proteinuria, incluso se ha visto como estos tratamientos pueden promover la regresión a normoalbuminuria (83).

En la nefropatía establecida (proteinuria), el tratamiento más eficaz se basa en IECA y ARA-II, que permiten frenar la progresión. La reducción de la PA disminuye la albuminuria y además enlentece la velocidad de descenso de la tasa de filtrado glomerular, aunque esto no se suele conseguir en fases avanzadas de la enfermedad. El control de la PA debe persistir en el tiempo para que sea eficaz. Además del descenso de la PA, la magnitud del descenso de la proteinuria en respuesta al tratamiento también es un factor independiente de protección renal (83).

Se recomiendan cifras de PA en el paciente diabético por debajo de 140/90 mm Hg para conseguir una nefro- protección eficaz, aunque si la proteinuria es superior a $1 \mathrm{~g} /$ día se debe intentar reducir aún más la PA a cifras algo inferiores a 130/80 mm Hg. De forma ideal, se debería reducir la magnitud de la albuminuria al menos un $50 \%$ e idealmente conseguir valores inferiores a $0,5-1 \mathrm{~g} /$ día en pacientes con ND. La magnitud de la albuminuria es además un marcador independiente de riesgo cardiovascular, incluso en el rango bajo de microalbuminuria, y, por ello, su normalización constituye un objetivo terapéutico. Para conseguir estos objetivos de reducción de PA y proteinuria, a menudo, es necesario añadir a los IECA o ARA-II otros antihipertensivos como calcioantagonistas (no dihidropiridínicos o dihidropiridínicos) y/o tiazidas o diuréticos de asa si la tasa de FG es inferior a $30 \mathrm{~mL} / \mathrm{min}$ y/o alfabloqueantes. Los betabloqueantes se deberían reservar para pacientes con una indicación adicional a usarlos, por ejemplo, en prevención secundaria de cardiopatía isquémica (83).

La restricción proteica está recomendada para frenar la progresión de la ND. Conforme avanza la insuficiencia renal, ha de ser compensada para evitar la desnutrición y el riesgo de hipercatabolismo, con un ajuste de la ingesta proteica a $0,6-0,8 \mathrm{~g} / \mathrm{kg}$ al día, con un $60 \%$ a $65 \%$ de hidratos de carbono, reducción de grasas saturadas a $1 / 3$ de las calorías totales y reducción de peso, especialmente en la DM2 (83).

Completan la protección de estos pacientes los tratamientos concomitantes, encaminados a controlar todos los factores que pueden influir en la progresión y el desarrollo de dicha complicación y en la morbimortalidad cardiovascular. Entre estos factores se encuentran la obe- 
sidad, el control de los lípidos con estatinas (objetivo de colesterol LDL inferior a $100 \mathrm{mg} / \mathrm{dL}$, HDL-colesterol superior a $45 \mathrm{mg} / \mathrm{dL}$ en varones y a $55 \mathrm{mg} / \mathrm{dL}$ en mujeres, triglicéridos por debajo de $150 \mathrm{mg} / \mathrm{dL}$ ), cese del hábito tabáquico y antiagregación plaquetaria en mayores de 45 años (83).

En presencia de insuficiencia renal crónica, la anemia es más precoz en el paciente diabético que en el no diabético y debe ser tratada tempranamente con agentes estimuladores de la eritropoyesis, dado que puede contribuir a mejorar la cardiopatía isquémica y a frenar la progresión de la hipertrofia ventricular izquierda y de la retinopatía, así como a mejorar la calidad de vida del paciente. En las fases avanzadas de insuficiencia renal se debe prevenir la malnutrición con dieta adecuada y suplementos. Cuando todas estas medidas fracasan y se avanza hacia la fase de ERCT, debe prepararse al paciente para su entrada en tratamiento sustitutivo renal. El paciente diabético puede ser candidato a un trasplante combinado renopancreático (en la DM1) o renal aislado (en la DM2), siempre bajo una estricta valoración del riesgo cardiovascular bien individualizada del paciente. La posibilidad de un trasplante de islotes pancreáticos aún no ofrece perspectivas exentas de un elevado riesgo (83).

\section{Referencias}

1. Organización Mundial de la Salud. Informe mundial sobre la diabetes [internet]. [Consultado 2017 Jun 02]. Disponible en: http://apps.who.int/iris/bitstream/10665/204877/1/WHO_NMH_ NVI_16.3_spa.pdf

2. Observatorio de Diabetes en Colombia [internet]. Bogotá: Organización para la Excelencia de la Salud OES. [Consultado 2017 Jun 02]. Disponible en: http://www.odc.org.co/

3. American diabetes association. Strategies for Improving Care. Diabetes care [internet] 2016;39(1):13-20 [Consultado 2017 Jun 02]. Disponible en: http://care.diabetesjournals.org/content/ supp1/2015/12/21/39.Supplement_1.DC2/2016-Standards-of-Care.pdf

4. Ibarra CT, Rocha J, Hernández R, Nieves RE, Leyva R. Prevalencia de neuropatía periférica en diabéticos tipo 2 en el primer nivel de atención. Rev Médica Chile. 2012;140(9):1126-1131.

5. Brownlee M, Aiello LP, Cooper ME, Vinik AI, Plutzky J, Boulton A. Complicaciones de la diabetes mellitus. En: Williams tratado de endocrinología. 13ed. España: Elsevier; 2017. p.1484-1581.

6. González CP. Monofilamento de Semmes-Weinstein. Diabetes Práctica Actual Habilidades En Aten Primaria. 2010;1(1):8-19.

7. Jurado J, Ybarra J, Romeo JH, Pou JM. Clinical screening and diagnosis of diabetic polyneuropathy: The North Catalonia Diabetes Study. Eur J Clin Invest. 2009;39(3):183-189.
8. Olmos PR, Niklitschek S, Olmos RI, Faúndez JI, Quezada TA, Bozinovic MA. Bases fisiopatológicas para una clasificación de la neuropatía diabética. Rev Médica Chile. 2012;140(12):15931605 .

9. Hernández BJ, Lluch MC, Nogueira JM. Fisiopatología. En: Tratado de pie diabético [internet]. España: Jarpyo Editores; 2012 [Consultado 2017 May 03]. Disponible en: http://www.sld.cu/galerias/pdf/sitios/rehabilitacion/capitulo_2.pdf

10. García WM. Neuropatía diabética: etiopatogenia y fisiopatologia. Medica hondureña [internet]. [2017 Mar 14]. Disponible en: http://www.bvs.hn/RMH/pdf/1984/pdf/Vol52-3-1984-6.pdf

11. Smith $G$, Updates in diabetic peripheral neuropathy [internet]. F1000 Faculty; 2016 [Consultado 2017 May 02]. Disponible en: https://www.ncbi.nlm.nih.gov/pmc/articles/PMC4847561/pdf/ f1000research-5-8502.pdf

12. Andrew JM, Boulton y Malik. Neuropathy En: Jameson, J. Larry. Endocrinology: Adult and Pediatric. 7ed. Philadelphia: Elsevier; 2016. p. $920-933$.

13. Pino F, Reynals de Blasis E, Vidal Puig A, Aschner Montoya P. Diabetes mellitus. En: Farreras Rozman Medicina Interna [internet]. 18 ed. Elsevier; 2016 [Consultado 2017 Feb 12]. p. 1824-1862. Disponible en: https://www.clinicalkey.es/\#!/content/book/3-s2.0B9788490229965002222? scrollTo $=\% 23 \mathrm{hl} 10002974$ (Farreras $\% 20$ Rozman.\%20Medicina\%20Interna\%20diabetes\%20mellitus)

14. Bakris G. Descripción general de la nefropatía diabética. [internet]. Wolters kluwer; 2016 [Consultado 2017 Feb 02]. Disponible en: https://www.uptodate.com/contents/overview-of-diabeticnephropathy?source=search_result\&search=nefropatia\%20diabet ica\&selectedTitle $=1 \sim 150 \#$ H6319416

15. López de Briña PE. Nefropatía diabética. En: Farreras Rozman Medicina Interna [internet]. 18 ed. Elsevier; 2016 [Consultado 2017 Feb 07]. p. 863-865. Disponible en: https://www.clinicalkey.es/\#!/content/book/3-s2.0-B978849022996500096X?scrollT $\mathrm{o}=\% 23 \mathrm{~h} 10000083$

16. Feldman E. Manifestaciones Clínicas y Diagnóstico de polineuropatia Diabética [internet]. Wolters kluwer; 2015 [Consultado 2017 Feb 12]. Disponible en: https://xa.yimg.com/kq/ groups/21983256/194883683/name/Clinical+manifestations+and + diagnosis + of + diabetic+polyneuropathy.pdf

17. Pradilla G. Diagnóstico y tratamiento de las neuropatías periféricas. [internet]. Asociacion Colombiana de Neurología; [Consultado 2017 Feb 12]. Disponible en: http://www.acnweb.org/guia/ g5cap14.pdf

18. Shy M. Neuropatías periféricas. En: Cecil y Goldman tratado de medicina interna [internet]. 24 ed. Madrid: Elsevier; 2013 [Consultado 2017 Feb 7]. Disponible en: https://www.clinicalkey. es/\#!/content/book/3-s2.0-B9788480869713004289

19. Ray T. Elevada frecuencia de neuropatía periférica en pacientes con Diabetes mellitus tipo 2 de un hospital general de LimaPerú. Rev Medica Hered [internet]. 2016 [Consultado 2017 Mar 05];24(2). Disponible en: http://www.perurevista.com/index.php/ medica/article/view/4566 
20. Yang Z, Chen R, Zhang Y, Huang Y, Hong T, Sun F, et al. Scoring systems to screen for diabetic peripheral neuropathy. En: The Cochrane Collaboration, editor. Cochrane Database of Systematic Reviews [internet]. Chichester, UK: John Wiley \& Sons, Ltd; 2014 [Consultado 2017 Mar 05]. Disponible en: http://doi.wiley. com/10.1002/14651858.CD010974

21. Herman WH, Pop-Busui R, Braffett BH, Martin CL, Cleary PA, Albers JW, et al. Use of the Michigan Neuropathy Screening Instrument as a measure of distal symmetrical peripheral neuropathy in Type 1 diabetes: results from the Diabetes Control and Complications Trial/Epidemiology of Diabetes Interventions and Complications: Michigan Neuropathy Screening Instrument in Type 1 diabetes. Diabet Med. 2012;29(7):937-944.

22. Katirji B. Neurología de Bradley en la práctica clínica. [internet]. 7. Vol. 1l. Elsevier; 2016 [Consultado 2017 Feb 12]. 1791-1866. p. Disponible en: https://www.clinicalkey.es/\#!/content/book/3s2.0-B9780323287838001071? scrollTo $=\% 23 \mathrm{~h} 10003626$

23. Feldman EL, McCulloch D. El tratamiento de la neuropatía diabética. [internet]. Wolters kluwer.; 2017 [Consultado 2017 Feb 12]. Disponible en: https://www.uptodate.com/ contents/treatment-of-diabetic-neuropathy? source=search result\&search $=$ neuropatia $\% 20$ diabetica\&selectedTitle $=1 \sim 150$

24. Botas Velasco M, Cervell Rodríguez D, Rodríguez Montalbán AI, Jiménez VS, Fernández de Valderrama Martínez I. Actualización en el diagnóstico, tratamiento y prevención de la neuropatía diabética periférica. Angiología [internet]. mayo de 2017 [Consultado 2017 Jun 02];69(3):174-81. Disponible en: http://linkinghub. elsevier.com/retrieve/pii/S0003317016300967.

25. San Miguel FV, Puente DM, Julià JV. Neuropatía diabética y pie diabético. Med-Programa Form Médica Contin Acreditado [internet]. 2016;12(17):971-981. [Consultado 2017 Jun 02]. Disponible en: http://www.sciencedirect.com/science/article/pii/ S0304541216301457

26. Isquemia miocárdica - MeSH - NCBI [internet]. Ncbi.nlm.nih. gov. [Consultado 2017 Feb 06]. Disponible en: https://www.ncbi. nlm.nih.gov/mesh/68017202

27. Moreno P, del Portillo J. Isquemia miocárdica: conceptos básicos, diagnóstico e implicaciones clínicas. Primera parte. Rev Colomb Cardiol. 2016;23(5):403-409

28. Organización Panamericana de la Salud. Perfil de Enfermedades Cardiovasculares. [internet]. [Consultado 2017 Feb 06]. Disponible en: http://www2.paho.org/hq/index.php?option=com_topic s\&view $=$ rdmore $\&$ cid $=7283 \&$ Itemid $=40876 \&$ lang $=$ es

29. Organización Mundial de la Salud. Enfermedades cardiovasculares [internet]. Who.int. 2015 [Consultado 2017 Feb 02]. Disponible en: http:/www.who.int/mediacentre/factsheets/fs317/es/

30. Organización Mundial de la Salud. Las 10 causas principales de defunción en el mundo [internet]. Who.int. 2014 [Consultado 2017 Feb 02]. Disponible en: http://www.who.int/mediacentre/ factsheets/fs310/es/

31. Marzilli M, Aterosclerosis coronaria obstructiva y cardiopatía isquémica: un eslabón elusivo! Elsevier [internet]. 2012;60(11):951956 [Consultado 2017 Feb 06] Disponible en: https://www.ncbi. nlm.nih.gov/pubmed/22954239
32. Puri R, Kataoka Y, Uno K, Nicholls S. La Naturaleza Distintiva de la Enfermedad Vascular Aterosclerótica en la Diabetes: Perspectivas Fisiopatológicas y Morfológicas. Informes actuales sobre la diabetes. 2012;12(3):280-285.

33. Zeadin M, Petlura C, Werstuck G. Mecanismos Moleculares Vinculando la Diabetes al Desarrollo Acelerado de la Aterosclerosis. Revista Canadiense de Diabetes. 2013;37(5):345-350.

34. Bornfeldt K. 2013 Russell Ross Conferencia Memorial en Biología Vascular: Mecanismos Celulares y Moleculares de la Diabetes Mellitus-Acelerada Aterosclerosis. Arterioesclerosis, Trombosis y Biología Vascular. 2014;34(4):705-714.

35. Moreno P, del Portillo J. Isquemia miocárdica: conceptos básicos, diagnóstico e implicaciones clínicas. Rev Colomb Cardiol. 2016;23(6):500-507.

36. Antman Loscalzo J. Cardiopatía isquémica. En: Loscalzo J, Kasper D, Fauci A, Hauser S, Longo D, Jameson J. Harrison Principios de Medicina Interna. 19 ed. Ciudad de México: McGrawHill; 2016. Página 1998-2015.

37. Flores Salinas H. Cardiovascular. En: Ramos Herrera I, Martínez Ceccopieri D, Hernández Chávez A, Centeno Flores M, Vázquez Valls R. CAM Curso de Actualización Medica [internet]. 2015 [Consultado 2017 Feb 10]. Disponible en: http://accessmedicina. mhmedical.com.recursosenlinea.juanncorpas.edu.co:2048/content.aspx?sectionid $=122466502$ \&bookid $=1739$ \& jumpsectionID $=$ $122466593 \&$ Resultclick=2\#1126986469

38. Bashore T, Granger C, Jackson K, Patel M. Cardiopatías. En: Papadakis McPhee S, Diagnóstico clínico y tratamiento. 54 ed. Madrid España: McGrawHill; 2016. p. 317-416.

39. Melo E, Cintra R, Biselli B, Melo R, Ribeiro H, Ávila L et al. Utilidad clínica de la angiografía coronaria y de la resonancia nuclear magnética en el diagnóstico de la cardiomiopatía isquémica. Rev Bras de Cardiol Invasiva. [internet]; 2013;21(3):276-80 [Consultado 2017 Feb 15]. Disponible en: http://www.scielo.br/scielo. php?script=sci_arttext\&pid=S2179-83972013000300014

40. Marui A. Cinco años de resultados de percutáneo frente a la cirugía coronaria de revascularización en pacientes con diabetes mellitus (de CREDO-Kyoto PCI/CABG Registry Cohort-2). Am J Cardiol. 2015;115(8):1063-1072.

41. Contini GA. Cinco años de resultados de revascularización miocárdica quirúrgica o percutánea en pacientes diabéticos. Int J Cardiol. 2013;168(2):1028-1033.

42. BARI 2D Study Group. Un ensayo aleatorio de los tratamientos para la diabetes tipo 2 y la enfermedad arterial coronaria. N Engl J Med. 2009;360(24):2503-2515.

43. Kappetein AP. Tratamiento de la enfermedad coronaria compleja en pacientes con diabetes: resultados a 5 años que comparan los resultados de la cirugía de bypass y la intervención coronaria percutánea en el estudio SYNTAX. Eur J Cardiothorac Surg. 2013;43(5):1006-1013.

44. Tcheugui E, Justin B. Sobre la importancia de la evaluación global del riesgo cardiovascular en personas con diabetes tipo 2 . Atención primaria Diabetes. 2014;7(2):95 - 102.

45. Tarquini, R., Lazzeri, C., Pala L. et al. La cardiomiopatía diabética. Acta Diabetol. 2011;48: paginas 173 - 180. 
46. Dandamudi. Prevalencia de la cardiopatía diabetica. Journal of Cardiac Failure. 2014; 20(5): 1015-1030.

47. Low Wang C, Hess C, Hiatt W, Goldfine A. Clinical Update: Enfermedad cardiovascular en diabetes. Circulation. 2016;133(24):2459-2502.

48. Jia, G, DeMarco VG, Sowers, JR. Resistencia a la insulina e hiperinsulinemia en cardiomiopatía diabética. Nature Reviews. Endocrinology. 2016;12(3):144-153.

49. McCormick, Liam M et al. Glucagon-like Peptide-1 protege contra la disfunción isquémica del ventrículo izquierdo durante la hiperglucemia en pacientes con enfermedad coronaria y diabetes mellitus tipo 2. Diabetología Cardiovascular. 2015:14(2):102110.

50. Mandavia $\mathrm{CH}$. Mecanismos moleculares y metabólicos de la disfunción cardíaca en la diabetes. Life Sciences. 2013: 92(18) página 601-608.

51. Joshi, M, Kotha SR, Malireddy S. Enigma de la patogénesis de la cardiomiopatía diabética: papel de la disfunción endotelial vascular, especies de oxígeno reactivo y mitocondrias. Mol Cell Biochem. 2014;36(27):1718-1727.

52. Seferović PM, Paulus WJ. Cardiomiopatía diabética clínica: una enfermedad de dos caras con fenotipos restrictivos y dilatados. Eur Heart J. 2015;36(27):1718-1727.

53. Yancy C, Jessup M, Bozkurt B, Butler J, Casey D, Drazner M et al. ACCF/AHA Guías para el manejo de la falla cardiaca. Rev Esp Cardiol. 2016;69:1167.

54. Guías para el diagnóstico y tratamiento de la insuficiencia cardíaca aguda y crónica 2012: El grupo de trabajo para el diagnóstico y tratamiento de la insuficiencia cardíaca aguda y crónica 2012 de la Sociedad Europea de Cardiología. Desarrollado en colaboración con la asociación de falla cardiaca (HFA) de la ESC. European Heart Journal. 2012;33(14):1787-1847.

55. Pérez del Villar C, Yotti R, Bermejo J. Técnicas de imagen en la insuficiencia cardiaca aguda. Revista Española de Cardiología. 2015;68(7) páginas: 612-623.

56. Fedele F, Gatto M, D’Ambrosi A, Mancone M. TNM-Like Clasificación: un nuevo método propuesto para la estadificación de la insuficiencia cardíaca. The Scientific World Journal. 2013:13(1):2028.

57. Fedele F, Severino P, Calcagno S, Mancone M. Heart Failure. J Am Coll Cardiol. 2014;63(19):1959-1960.

58. Rozman Borstnar C, Cardellach F. Farreras Rozman. Medicina interna [internet]. Barcelona: Elsevier; 2016. Páginas 863-865 [Consultado 2017 May 31]. Disponible en: https:// www.clinicalkey.es/service/content/pdf/watermarked/3-s2.0B978849022996500096X.pdf?locale=es_ES

59. Cecil RL, Goldman L, Schafer AI. Nefropatías hereditarias y alteraciones del tracto urinario en Goldman and Cecil. 25ed. Barcelona: Elsevier; 2016. 804-806.

60. Gonzalez Suarez ML, Thomas DB, Barisoni L, Fornoni A. Diabetic nephropathy: Is it time yet for routine kidney biopsy? World J Diabetes. 2013;4:245-255.
61. Eggers PW. Has the incidence of end-stage renal disease in the USA and other countries stabilized? Curr Opin Nephrol Hypertens. 2012;20:241-245.

62. Diez RG, Gonzalez Bedat M, Pecoits Filho R, Marinovich S, Fernandez S, Lugon J, et al. Renal replacement therapy in Latin American end-stage renal disease. Clin Kidney J. 2014;7:431-436.

63. Fried LF, Emanuele N, Zhang JH, Brophy M, Conner TA, Duckworth W, et al. Combined angiotensin inhibition for the treatment of diabetic nephropathy. N Engl J Med. 2013;369(14):1892-1903.

64. Roscioni SS, Heerspink HJ, de Zeeuw D. The effect of RAAS blockade on the progression of diabetic nephropathy. Nat Rev Nephrol. 2014;10:77-87.

65. Ministerio de la Protección Social, Programa de Apoyo a la Reforma de Salud. Guía para el manejo de la enfermedad renal crónica y modelo de prevención y control de la enfermedad renal crónica. Componente de un modelo de salud renal. Bogotá: Ministerio de la Protección Social; 2007.

66. Martínez F, Ordóñez I, García D. Deficiencias en el tratamiento de pacientes diabéticos que terminaron en enfermedad renal crónica. Acta Médica Colombiana. 2007; 32(2) páginas: 135-150.

67. Gómez RA. Renal disease in Colombia. Renal Failure. 2006;28(8):643-647.

68. Alcázar R, Portolés JM, Egocheaga I, Lobos JM, Rosado J, Arrieta $\mathrm{F}$, et al. Recomendaciones sobre la detección, seguimiento y criterios de derivación de la enfermedad renal crónica en el ámbito de la Comunidad de Madrid. Médicos de familia. 2012(1);13:916.

69. Gómez Huelgas R, Martínez Castelao A, Artola S, Górriz JL, Menéndez E. Grupo de trabajo para el documento de consenso sobre el tratamiento de la diabetes tipo 2 en el paciente con enfermedad renal crónica. Nefrologia. 2014; 34(1):34-45.

70. De Briñas, López EP. Nefropatía diabética. En: Farreras Rozman. Medicina Interna 19ed. España. Elsevier; 2016. p. 863-865.

71. Kelly KJ, Dominguez JH. Treatment of the post-ischaemic inflammatory syndrome of diabetic nephropathy. Nephrol Dial Transplant Off Publ Eur Dial Transpl Assoc - Eur Ren Assoc. octubre de 2012; 25(10):3204-3212.

72. Campbell KN, Raij L, Mundel P. Role of angiotensin II in the development of nephropathy and podocytopathy of diabetes. Curr Diabetes Rev. 2012;7(1):3-50.

73. Obrador Vera GT. Enfermedad renal crónica. En: Farreras Rozman. Medicina Interna 19ed. España. Elsevier; 2016. p. 829-836.

74. Carranza K, Veron D, Cercado A, Bautista N, Pozo W, Tufro A, et al. Aspectos celulares y moleculares de la nefropatía diabética, rol del VEGF-A. Nefrología. 2015;35(2):131-138.

75. Bakris G, Glassock R, Nathan M, Descripción general de la nefropatía diabética [internt]. [Consultado $2017 \mathrm{Feb}$ 18]. https:// www-uptodate-com.recursosenlinea.juanncorpas.edu.co:2443/ contents/overview-of-diabetic-nephropathy? source $=$ search result\&search=nefropatía\%20diabética\&selectedTitle $=1 \sim 15 \overline{0}$ \#H6319416 
76. Rossing P, Fioretto P, Rasmussen B, Parving HH. Diabetic Nephropathy. Brenner and Rector's. The Kidney 2016:39(9):1283-1321

77. Masharani U. Diabetes mellitus e hipoglucemia. In: Papadakis MA, McPhee SJ, Rabow MW. eds. Diagnóstico clínico y tratamiento. 54ed. New York, NY: McGraw-Hill; 2015. http://accessmedicina.mhmedical.com.recursosenlinea.juanncorpas.edu. co:2048/content.aspx ?bookid $=1610 \&$ sectionid $=99862701$. Accessed marzo 05, 2017.

78. Improving Global Outcomes (KDIGO) CKD Work Group. KDIGO Clinical Practice Guideline for the Evaluation and Management of Chronic Kidney Disease. Kidney Int Suppl 2013;3(1):6-308.

79. Otero A, de Francisco A, Gayoso P, García F; on behalf of the EPIRCE Study Group. Prevalence of chronic renal disease in Spain: results of the EPIRCE study. Nefrologia. 2012;30(1):78-86.

80. Martínez Castelao A, Górriz JL, Bover J, Segura-de la Morena J, Cebollada J, Escalada J, et al. Documento de consenso para la de- tección y manejo de la enfermedad renal crónica. Aten Primaria. 2014;46(9):501-519.

81. Levey AS, Stevens LA, Schmid CH, Zhang YL, Castro AF 3rd, Feldman HI, et al.; CKD-EPI (Chronic Kidney Disease Epidemiology Collaboration). A new equation to estimate glomerular filtration rate. Ann Intern Med. 2009;150:604-612.

82. Medavilla bravo. Guías clínicas diabetes mellitus. [internet] Semergen. 2015, págs. 132-145 [Consultado 2017 Jun 01]. Disponible en: http://2016.jornadasdiabetes.com/docs/Guia_Diabetes_Semergen.pdf

83. Rojas Guzman, Gracia Rubí. Prevención, diagnóstico y tratamiento temprano de la Nefropatía Diabética. Recomendaciones de la Asociación Latinoamericana de Diabetes (ALAD) Avalado por la Sociedad Latinoamericana de Nefrología e Hipertensión (SLANH) [internet]. [Consultado 2017 Jun 01]. Disponible en: http://alad-americalatina.org/wp-content/uploads/2016/10/PREVENCION-DE-NEFROPATIA.pdf 